\title{
Multipliers on $\mathcal{S}_{\omega}\left(\mathbb{R}^{N}\right)$
}

\author{
Angela A. Albanese ${ }^{1}$ (D) $\cdot$ Claudio Mele $^{1}$
}

Received: 22 March 2021 / Revised: 7 April 2021 / Accepted: 12 April 2021 / Published online: 24 May 2021

(c) The Author(s) 2021

\section{Abstract}

The aim of this paper is to introduce and to study the space $\mathcal{O}_{M, \omega}\left(\mathbb{R}^{N}\right)$ of the multipliers of the space $\mathcal{S}_{\omega}\left(\mathbb{R}^{N}\right)$ of the $\omega$-ultradifferentiable rapidly decreasing functions of Beurling type. We determine various properties of the space $\mathcal{O}_{M, \omega}\left(\mathbb{R}^{N}\right)$. Moreover, we define and compare some lc-topologies of which $\mathcal{O}_{M, \omega}\left(\mathbb{R}^{N}\right)$ can be naturally endowed.

Keywords Multipliers · Weight functions · Ultradifferentiable rapidly decreasing function spaces of Beurling type

Mathematics Subject Classification Primary 46E10 - 46F05; Secondary 47B38

\section{Introduction}

Classes of ultradifferentiable functions have been intensively investigated since the 20ies of the last century. The subject indeed has a long tradition that goes back to the work of Gevrey [11]. Along the lines of [11], Komatsu introduced in [16] a way to define the ultradifferentiable functions. It consists in measuring their growth behaviour in terms of a weight sequence $\left\{M_{p}\right\}_{p \in \mathbb{N}_{0}}$, which satisfies certain conditions. Later Beurling [1] (see also Björck [2]) showed that one can also use weight functions $\omega$ to measure the smoothness of $C^{\infty}$-functions with compact support by the decay properties of their Fourier transform. This approach was modified by Braun, Meise, and Taylor [8], who showed that these classes can be defined by the decay behaviour of their derivatives by using the Young conjugate of the function $t \mapsto \omega\left(e^{t}\right)$. But, in

Angela A. Albanese

angela.albanese@unisalento.it

Claudio Mele

claudio.mele1@unisalento.it

1 Dipartimento di Matematica e Fisica “E. De Giorgi”, Università del Salento, C.P.193, 73100 Lecce, Italy 
general, the classes defined in one way cannot be defined in the other way (see, f.i., [6]).

The study of the space of multipliers and convolutors, $\mathcal{O}_{M}$ and $\mathcal{O}_{C}^{\prime}$, of the space $\mathcal{S}\left(\mathbb{R}^{N}\right)$ of rapidly decreasing functions was started by Schwartz [27]. Since then the spaces $\mathcal{O}_{M}$ and $\mathcal{O}_{C}^{\prime}$ attracted the attention of several authors, even recently (see [15,19$22,24]$ and the references therein). On the other hand, the space of multipliers and convolutors was introduced and studied in the setting of other classes of $C^{\infty}$-function spaces (see, f.i., [10,14,18,25,26,28,29] and the references therein).

In the last years the attention has focused on the study of the space $\mathcal{S}_{\omega}\left(\mathbb{R}^{N}\right)$ of the ultradifferentiable rapidly decreasing functions of Beurling type, as introduced by Björck [2] (see, [3-5], for instance, and the references therein). Inspired by this line of research and by the previous work, in this paper we introduce and study the space $\mathcal{O}_{M, \omega}\left(\mathbb{R}^{N}\right)$ of the slowly increasing functions of Beurling type in the setting of ultradifferentiable function space as introduced in [8]. In particular, we show that $\mathcal{O}_{M, \omega}\left(\mathbb{R}^{N}\right)$ is the space of the multipliers of the space $\mathcal{S}_{\omega}\left(\mathbb{R}^{N}\right)$ and of its dual $\mathcal{S}_{\omega}^{\prime}\left(\mathbb{R}^{N}\right)$. We also define and compare some locally convex topologies (briefly, lc) of which $\mathcal{O}_{M, \omega}\left(\mathbb{R}^{N}\right)$ can be naturally endowed.

The paper is organized as follows. Section 2 collects some known definitions and properties about the weight functions in the sense of Braun, Meise and Taylor [8] and the space $\mathcal{S}_{\omega}\left(\mathbb{R}^{N}\right)$. Section 3 is devoted to the study of the space $\mathcal{O}_{M, \omega}\left(\mathbb{R}^{N}\right)$ and of related topological properties. In Sect. 3 we also introduce the space $\mathcal{O}_{C, \omega}\left(\mathbb{R}^{N}\right)$ of the very slowly increasing functions of Beurling type. In particular, we show the link between these spaces and their topological properties. In a forthcoming paper we prove, f.i., that $\mathcal{O}_{C, \omega}^{\prime}\left(\mathbb{R}^{N}\right)$ is the space of convolutors of $\mathcal{S}_{\omega}^{\prime}\left(\mathbb{R}^{N}\right)$ and the Fourier exchange between the spaces $\mathcal{O}_{C, \omega}^{\prime}\left(\mathbb{R}^{N}\right)$ and $\mathcal{O}_{M, \omega}\left(\mathbb{R}^{N}\right)$. In Sect. 4 we show that $\mathcal{O}_{M, \omega}\left(\mathbb{R}^{N}\right)$ is the space of the multipliers of $\mathcal{S}_{\omega}\left(\mathbb{R}^{N}\right)$. Finally, in Sect. 5 we study and compare some lc-topologies of which $\mathcal{O}_{M, \omega}\left(\mathbb{R}^{N}\right)$ can be endowed in a natural way.

\section{Weight functions and the space $\mathcal{S}_{\omega}\left(\mathbb{R}^{N}\right)$}

We begin with the definition of non-quasianalytic weight function in the sense of Braun-Meise-Taylor [8] suitable for the Beurling case, i.e., we also consider the logarithm as a weight function.

Definition 2.1 A non-quasianalytic weight function is a continuous increasing function $\omega:[0, \infty) \rightarrow[0, \infty)$ satisfying the following properties:

$(\alpha)$ there exists $K \geq 1$ such that $\omega(2 t) \leq K(1+\omega(t))$ for every $t \geq 0$;

( $\beta) \int_{1}^{\infty} \frac{\omega(t)}{1+t^{2}} d t<\infty$

$(\gamma)$ there exist $a \in \mathbb{R}, b>0$ such that $\omega(t) \geq a+b \log (1+t)$, for every $t \geq 0$;

( $\delta) \varphi_{\omega}(t)=\omega \circ \exp (t)$ is a convex function.

Given a non-quasianalytic weight function $\omega$, we can extend it on $\mathbb{C}^{N}$ by setting $\omega(z)=\omega(|z|)$ for all $z \in \mathbb{C}^{N}$, where $|\cdot|$ denotes the standard euclidean norm. 
Remark 2.2 We now recall some known facts on weight functions that shall be useful in the following; the proofs can be found in the literature.

Let $\omega$ be a non-quasianalytic weight function. Then the following properties are satisfied.

(1) Condition $(\alpha)$ implies for every $t_{1}, t_{2} \geq 0$ that

$$
\omega\left(t_{1}+t_{2}\right) \leq K\left(1+\omega\left(t_{1}\right)+\omega\left(t_{2}\right)\right)
$$

Observe that this condition is weaker than subadditivity (i.e., $\omega\left(t_{1}+t_{2}\right) \leq \omega\left(t_{1}\right)+$ $\left.\omega\left(t_{2}\right)\right)$. The weight functions satisfying $(\alpha)$ are not necessarily subadditive in general.

(2) Condition $(\alpha)$ implies that there exists $L \geq 1$ such that for every $t \geq 0$

$$
\omega(e t) \leq L(1+\omega(t))
$$

(3) By condition $(\beta)$ and the fact that $\omega$ is an increasing function, we have that $\omega(t)=o(t)$ as $t \rightarrow \infty$. This can be deduced by the fact that for every $t>0$ we have

$$
\frac{\omega(t)}{t}=\int_{t}^{\infty} \frac{\omega(t)}{s^{2}} d s \leq \int_{t}^{\infty} \frac{\omega(s)}{s^{2}} d s
$$

Let $\omega$ be a non-quasianalytic weight function. We define the Young conjugate $\varphi_{\omega}^{*}$ of $\varphi_{\omega}$ as the function $\varphi_{\omega}^{*}:[0, \infty) \rightarrow[0, \infty)$ defined by

$$
\varphi_{\omega}^{*}(s):=\sup _{t \geq 0}\left\{s t-\varphi_{\omega}(t)\right\}, \quad s \geq 0 .
$$

There is no loss of generality to assume that $\omega$ vanishes on $[0,1]$. So, $\varphi_{\omega}^{*}$ has only non-negative values. By Fenchel-Moreau Theorem (see, f.i., [7]), we have that $\varphi_{\omega}^{*}$ is convex and increasing, $\varphi_{\omega}^{*}(0)=0$ and $\left(\varphi_{\omega}^{*}\right)^{*}=\varphi_{\omega}$. Further useful properties of $\varphi_{\omega}^{*}$ are listed in the following lemma, see [8].

Lemma 2.3 Let $\omega:[0, \infty) \rightarrow[0, \infty)$ be a non-quasianalytic weight function. Then the following properties are satisfied.

(1) $\lim _{t \rightarrow \infty} \frac{t}{\varphi_{\omega}^{*}(t)}=0$.

(2) $\frac{\varphi_{\omega}^{*}(t)}{t}$ is an increasing function in $(0, \infty)$.

(3) For every $s, t \geq 0$ and $\lambda>0$

$$
2 \lambda \varphi_{\omega}^{*}\left(\frac{s+t}{2 \lambda}\right) \leq \lambda \varphi_{\omega}^{*}\left(\frac{s}{\lambda}\right)+\lambda \varphi_{\omega}^{*}\left(\frac{t}{\lambda}\right) \leq \lambda \varphi_{\omega}^{*}\left(\frac{s+t}{\lambda}\right) .
$$

(4) For every $t \geq 0$ and $\lambda>0$

$$
\lambda L \varphi_{\omega}^{*}\left(\frac{t}{\lambda L}\right)+t \leq \lambda \varphi_{\omega}^{*}\left(\frac{t}{\lambda}\right)+\lambda L
$$

where $L \geq 1$ is the costant appearing in formula (2.2). 
Remark 2.4 Lemma 2.3 allows to list some properties that we will frequently use in the sequel.

(1) For every $m, M \in \mathbb{N}$ with $M \geq m L$, where $L$ is the constant appearing in formula (2.2), and for every $\alpha \in \mathbb{N}_{0}^{N}$

$$
2^{|\alpha|} \exp \left(M \varphi_{\omega}^{*}\left(\frac{|\alpha|}{M}\right)\right) \leq C \exp \left(m \varphi_{\omega}^{*}\left(\frac{|\alpha|}{m}\right)\right)
$$

with $C:=e^{m L}$.

(2) For every $\alpha, \beta \in \mathbb{N}_{0}^{N}$ and $m \in \mathbb{N}$

$$
2 m \varphi_{\omega}^{*}\left(\frac{|\alpha+\beta|}{2 m}\right) \leq m \varphi_{\omega}^{*}\left(\frac{|\alpha|}{m}\right)+m \varphi_{\omega}^{*}\left(\frac{|\beta|}{m}\right) \leq m \varphi_{\omega}^{*}\left(\frac{|\alpha+\beta|}{m}\right) .
$$

We now introduce the ultradifferentiable function spaces and their duals of Beurling type in the sense of Braun, Meise and Taylor [8].

Definition 2.5 Let $\omega$ be a non-quasianalytic weight.

(a) For a compact subset $K$ of $\mathbb{R}^{N}$ and $\lambda>0$ define

$$
\mathcal{E}_{\omega, \lambda}(K):=\left\{f \in C^{\infty}(K): p_{K, \lambda}(f):=\sup _{x \in K} \sup _{\alpha \in \mathbb{N}_{0}^{N}}\left|\partial^{\alpha} f(x)\right| \exp \left(-\lambda \varphi_{\omega}^{*}\left(\frac{|\alpha|}{\lambda}\right)\right)<\infty\right\} .
$$

Then $\left(\mathcal{E}_{\omega, \lambda}(K), p_{K, \lambda}\right)$ is a Banach space.

(b) For an open subset $\Omega$ of $\mathbb{R}^{N}$ define

$$
\mathcal{E}_{\omega}(\Omega):=\left\{f \in C^{\infty}(\Omega): p_{K, m}(f)<\infty \forall K \Subset \Omega, m \in \mathbb{N}\right\}
$$

and endow it with its natural Fréchet space topology, i.e., with the lc-topology generated by the system of seminorms $\left\{p_{K, m}\right\}_{K \Subset \Omega, m \in \mathbb{N}}$. The elements of $\mathcal{E}_{\omega}(\Omega)$ are called $\omega$-ultradifferentiable functions of Beurling type on $\Omega$. The dual $\mathcal{E}_{\omega}^{\prime}(\Omega)$ of $\mathcal{E}_{\omega}(\Omega)$ is endowed with its strong topology.

(c) For a compact subset $K$ of $\mathbb{R}^{N}$ define

$$
\mathcal{D}_{\omega}(K):=\left\{f \in \mathcal{E}_{\omega}\left(\mathbb{R}^{N}\right): \operatorname{supp} f \subseteq K\right\}
$$

and endow it with the Fréchet space topology generated by the sequence $\left\{p_{K, m}\right\}_{m \in \mathbb{N}}$ of norms. For an open subset $\Omega$ of $\mathbb{R}^{N}$ define

$$
\mathcal{D}_{\omega}(\Omega):=\text { ind }{ }_{j \rightarrow} \mathcal{D}_{\omega}\left(K_{j}\right)
$$

where $\left\{K_{j}\right\}_{j \in \mathbb{N}}$ is any fundamental sequence of compact subsets of $\Omega$. The elements of $\mathcal{D}_{\omega}(\Omega)$ are called test functions of Beurling type on $\Omega$. The dual $\mathcal{D}_{\omega}^{\prime}(\Omega)$ of $\mathcal{D}_{\omega}(\Omega)$ is endowed with its strong topology. The elements of $\mathcal{D}_{\omega}^{\prime}(\Omega)$ are called $\omega$-ultradistributions of Beurling type on $\Omega$. 
Remark 2.6 Let $\omega$ be a non-quasianalytic weight function. Then the following properties are satisfied.

(1) For every open subset $\Omega$ of $\mathbb{R}^{N}$ the space $\mathcal{E}_{\omega}(\Omega)$ is a nuclear Fréchet space, see [8, Proposition 4.9].

(2) For every compact subset $K$ of $\mathbb{R}^{N}$ we have $\mathcal{D}_{\omega}(K) \neq\{0\}$ and $\mathcal{D}_{\omega}(K)$ is a nuclear Fréchet space, see [8, Remark 3.2 (1) and Corollary 3.6(2)].

(3) For every open subset $\Omega$ of $\mathbb{R}^{N}$ the inclusion $\mathcal{D}_{\omega}(\Omega) \hookrightarrow \mathcal{E}_{\omega}(\Omega)$ is continuous with dense range, see [8, Proposition 4.7 (1)].

We consider the following notation for the Fourier transform of a function $f \in$ $L^{1}\left(\mathbb{R}^{N}\right)$ :

$$
\mathcal{F}(f)(\xi)=\hat{f}(\xi):=\int_{\mathbb{R}^{N}} f(x) e^{-i x \xi} d x, \quad \xi \in \mathbb{R}^{N},
$$

with standard extensions to more general spaces of functions or distributions. We introduce the space of weighted rapidly decreasing functions of Beurling type as defined in [2, Definition 1.8.1].

Definition 2.7 Let $\omega$ be a non-quasianalytic weight function. We denote by $\mathcal{S}_{\omega}\left(\mathbb{R}^{N}\right)$ the set of all functions $f \in L^{1}\left(\mathbb{R}^{N}\right)$ such that $f, \hat{f} \in C^{\infty}\left(\mathbb{R}^{N}\right)$ and for each $\lambda>0$ and $\alpha \in \mathbb{N}_{0}^{N}$ we have

$$
\left\|\exp (\lambda \omega) \partial^{\alpha} f\right\|_{\infty}<\infty \text { and }\left\|\exp (\lambda \omega) \partial^{\alpha} \hat{f}\right\|_{\infty}<\infty
$$

The elements of $\mathcal{S}_{\omega}\left(\mathbb{R}^{N}\right)$ are called $\omega$-ultradifferentiable rapidly decreasing functions of Beurling type.

The space $\mathcal{S}_{\omega}\left(\mathbb{R}^{N}\right)$ is a Fréchet space with respect to the lc-topology generated by the sequence of norms

$$
\left\|\exp (n \omega) \partial^{\alpha} f\right\|_{\infty}+\left\|\exp (n \omega) \partial^{\alpha} \hat{f}\right\|_{\infty}, \quad f \in \mathcal{S}_{\omega}\left(\mathbb{R}^{N}\right), n \in \mathbb{N} .
$$

We denote by $\mathcal{S}_{\omega}^{\prime}\left(\mathbb{R}^{N}\right)$ the dual of $\mathcal{S}_{\omega}\left(\mathbb{R}^{N}\right)$ endowed with its strong topology.

Remark 2.8 Let $\omega$ be a non-quasianalytic weight function. Then the following properties are satisfied.

(1) The condition $(\gamma)$ of Definition 2.1 implies that $\mathcal{S}_{\omega}\left(\mathbb{R}^{N}\right) \subseteq \mathcal{S}\left(\mathbb{R}^{N}\right)$ with continuous inclusion. Accordingly, we can rewrite the definition of $\mathcal{S}_{\omega}\left(\mathbb{R}^{N}\right)$ as the set of all the rapidly decreasing functions that satisfy the condition (2.8) in Definition 2.7 .

(2) The Fourier transform $\mathcal{F}: \mathcal{S}_{\omega}\left(\mathbb{R}^{N}\right) \rightarrow \mathcal{S}_{\omega}\left(\mathbb{R}^{N}\right)$ is a continuous isomorphism, that can be extended in the usual way to $\mathcal{S}_{\omega}^{\prime}\left(\mathbb{R}^{N}\right)$, see [2, Proposition 1.8.2].

(3) The space $\mathcal{S}_{\omega}\left(\mathbb{R}^{N}\right)$ is closed under convolution, under multiplication, translation and modulation, where the translation and modulation operators are defined by $\tau_{y} f(x):=f(x-y)$ and $M_{t} f(x):=e^{i t x} f(x)$, respectively, where $t, x, y \in \mathbb{R}^{N}$, see [2, Propositions 1.8.3 and 18.5]. 
(4) The inclusion $\mathcal{D}_{\omega}\left(\mathbb{R}^{N}\right) \hookrightarrow \mathcal{S}_{\omega}\left(\mathbb{R}^{N}\right) \hookrightarrow \mathcal{E}_{\omega}\left(\mathbb{R}^{N}\right)$ are continuous with dense range, see [8, Proposition 4.7.(1)] and [2, Propositions 1.8.6 and 1.8.7]. Therefore, $\mathcal{E}_{\omega}^{\prime}\left(\mathbb{R}^{N}\right) \subset \mathcal{S}_{\omega}^{\prime}\left(\mathbb{R}^{N}\right) \subset \mathcal{D}_{\omega}^{\prime}\left(\mathbb{R}^{N}\right)$.

(5) The space $\mathcal{S}_{\omega}\left(\mathbb{R}^{N}\right)$ is a nuclear Fréchet space, see [5, Theorem 3.3].

The space $\mathcal{S}_{\omega}\left(\mathbb{R}^{N}\right)$ is a Fréchet space with different equivalent systems of seminorms. Indeed, the following result holds (see [4, Theorem 4.8] and [3, Theorems 2.6]).

Proposition 2.9 Let $\omega$ be a non-quasianalytic weight function and consider $f \in$ $\mathcal{S}\left(\mathbb{R}^{N}\right)$. Then $f \in \mathcal{S}_{\omega}\left(\mathbb{R}^{N}\right)$ if and only if one of the following conditions is satisfied.

(1) (i) $\forall \lambda>0, \alpha \in \mathbb{N}_{0}^{N}, 1 \leq p \leq \infty \exists C_{\alpha, \lambda, p}>0$ such that $\left\|\exp (\lambda \omega) \partial^{\alpha} f\right\|_{p} \leq$ $C_{\alpha, \lambda, p}$, and

(ii) $\forall \lambda>0, \alpha \in \mathbb{N}_{0}^{N}, 1 \leq p \leq \infty \exists C_{\alpha, \lambda, p}>0$ such that $\left\|\exp (\lambda \omega) \partial^{\alpha} \hat{f}\right\|_{p} \leq$ $C_{\alpha, \lambda, p}$

(2) (i) $\forall \lambda>0,1 \leq p \leq \infty \exists C_{\lambda, p}>0$ such that $\|\exp (\lambda \omega) f\|_{p} \leq C_{\lambda, p}$, and

(ii) $\forall \lambda>0,1 \leq p \leq \infty \exists C_{\lambda, p}>0$ such that $\|\exp (\lambda \omega) \hat{f}\|_{p} \leq C_{\lambda, p}$.

(3) $\forall \lambda, \mu>0,1 \leq p \leq \infty \exists C_{\lambda, \mu, p}>0$ such that

$$
\sup _{\alpha \in \mathbb{N}_{0}^{N}}\left\|\exp (\mu \omega) \partial^{\alpha} f\right\|_{p} \exp \left(-\lambda \varphi_{\omega}^{*}\left(\frac{|\alpha|}{\lambda}\right)\right) \leq C_{\lambda, \mu, p}
$$

Remark 2.10 We observe that the assumption $f \in \mathcal{S}\left(\mathbb{R}^{N}\right)$ in Proposition 2.9 can be replaced by the weaker assumption $f \in C^{\infty}\left(\mathbb{R}^{N}\right)$. Indeed, the condition $(\gamma)$ in Definition 2.1 implies for every $x \in \mathbb{R}^{N}$ and $\alpha \in \mathbb{N}_{0}^{N}$ that

$$
\left|x^{\alpha}\right| \leq \exp \left(-\frac{|\alpha|}{b}\right) \exp \left(\frac{|\alpha|}{b} \omega(x)\right)
$$

where $b$ is the constant appearing in condition $(\gamma)$. Therefore, if one (and hence all) of the equivalent conditions $(1) \div(3)$ of Proposition 2.9 is satisfied, then for every $\alpha, \beta \in \mathbb{N}_{0}^{N}$ and $1 \leq p \leq \infty$ we have

$$
\left\|x^{\alpha} \partial^{\beta} f\right\|_{p} \leq \exp \left(-\frac{|\alpha|}{b}\right)\left\|\exp \left(\frac{|\alpha|}{b} \omega\right) \partial^{\beta} f\right\|_{p}<\infty \text {. }
$$

Accordingly, $f \in \mathcal{S}\left(\mathbb{R}^{N}\right)$.

In the following, we will use this system of norms generating the Fréchet topology of $\mathcal{S}_{\omega}\left(\mathbb{R}^{N}\right)$ :

$$
q_{\lambda, \mu}(f):=\sup _{\alpha \in \mathbb{N}_{0}^{N}} \sup _{x \in \mathbb{R}^{N}} \exp \left(-\lambda \varphi_{\omega}^{*}\left(\frac{|\alpha|}{\lambda}\right)\right) \exp (\mu \omega(x))\left|\partial^{\alpha} f(x)\right|, \lambda, \mu>0, f \in \mathcal{S}_{\omega}\left(\mathbb{R}^{N}\right) .
$$

In particular, the Fréchet topology of $\mathcal{S}_{\omega}\left(\mathbb{R}^{N}\right)$ is generated by the sequence of norms $\left\{q_{m, n}\right\}_{m, n \in \mathbb{N}}$. 


\section{The spaces $\mathcal{O}_{M, \omega}\left(\mathbb{R}^{N}\right)$ and $\mathcal{O}_{C, \omega}\left(\mathbb{R}^{N}\right)$}

We first introduce the spaces $\mathcal{O}_{M, \omega}\left(\mathbb{R}^{N}\right)$ and $\mathcal{O}_{C, \omega}\left(\mathbb{R}^{N}\right)$ and then we collect some basic properties about them.

Definition 3.1 Let $\omega$ be a non-quasianalytic weight function.

(a) For $m \in \mathbb{N}$ and $n \in \mathbb{Z}$ we define the space $\mathcal{O}_{n, \omega}^{m}\left(\mathbb{R}^{N}\right)$ as the set of all functions $f \in C^{\infty}\left(\mathbb{R}^{N}\right)$ satisfying the following condition:

$$
r_{m, n}(f):=\sup _{\alpha \in \mathbb{N}_{0}^{N}} \sup _{x \in \mathbb{R}^{N}}\left|\partial^{\alpha} f(x)\right| \exp \left(-n \omega(x)-m \varphi_{\omega}^{*}\left(\frac{|\alpha|}{m}\right)\right)<\infty .
$$

(b) We denote by $\mathcal{O}_{M, \omega}\left(\mathbb{R}^{N}\right)$ the set of all functions $f \in C^{\infty}\left(\mathbb{R}^{N}\right)$ such that for each $m \in \mathbb{N}$ there exist $C>0$ and $n \in \mathbb{N}$ such that for every $\alpha \in \mathbb{N}_{0}^{N}$ and $x \in \mathbb{R}^{N}$ we have

$$
\left|\partial^{\alpha} f(x)\right| \leq C \exp \left(n \omega(x)+m \varphi_{\omega}^{*}\left(\frac{|\alpha|}{m}\right)\right)
$$

or equivalently,

$$
\mathcal{O}_{M, \omega}\left(\mathbb{R}^{N}\right):=\bigcap_{m=1}^{\infty} \bigcup_{n=1}^{\infty} \mathcal{O}_{n, \omega}^{m}\left(\mathbb{R}^{N}\right)
$$

The elements of $\mathcal{O}_{M, \omega}\left(\mathbb{R}^{N}\right)$ are called slowly increasing functions of Beurling type.

(c) We denote by $\mathcal{O}_{C, \omega}\left(\mathbb{R}^{N}\right)$ the set of all functions $f \in C^{\infty}\left(\mathbb{R}^{N}\right)$ for which there exists $n \in \mathbb{N}$ such that for every $m \in \mathbb{N}$ there exists $C>0$ so that for every $\alpha \in \mathbb{N}_{0}^{N}$ and $x \in \mathbb{R}^{N}$ we have

$$
\left|\partial^{\alpha} f(x)\right| \leq C \exp \left(n \omega(x)+m \varphi_{\omega}^{*}\left(\frac{|\alpha|}{m}\right)\right)
$$

or equivalently,

$$
\mathcal{O}_{C, \omega}\left(\mathbb{R}^{N}\right):=\bigcup_{n=1}^{\infty} \bigcap_{m=1}^{\infty} \mathcal{O}_{n, \omega}^{m}\left(\mathbb{R}^{N}\right)
$$

The elements of $\mathcal{O}_{C, \omega}\left(\mathbb{R}^{N}\right)$ are called very slowly increasing functions of Beurling type.

Remark 3.2 By Definition 3.1, we immediately obtain the following properties.

(1) $\mathcal{D}_{\omega}\left(\mathbb{R}^{N}\right) \subseteq \mathcal{O}_{C, \omega}\left(\mathbb{R}^{N}\right)$ and $\mathcal{D}_{\omega}\left(\mathbb{R}^{N}\right) \subseteq \mathcal{O}_{M, \omega}\left(\mathbb{R}^{N}\right)$.

(2) For every $\alpha \in \mathbb{N}_{0}^{N}$ the function $x^{\alpha} \in \mathcal{O}_{M, \omega}\left(\mathbb{R}^{N}\right)$. Indeed, fixed any $\alpha \in \mathbb{N}_{0}^{N}$, we have that $\partial^{\beta} x^{\alpha}=\prod_{i=1}^{N} \alpha_{i}\left(\alpha_{i}-1\right) \ldots\left(\alpha_{i}-\beta_{i}+1\right) x_{i}^{\alpha_{i}-\beta_{i}}$ for $\beta \leq \alpha$, and that 
$\partial^{\beta} x^{\alpha}=0$ for $\beta>\alpha$. Since condition $(\gamma)$ implies that $(1+t) \leq e^{-\frac{a}{b}} e^{\frac{1}{b} \omega(t)}$ for all $t \geq 0$ and $\omega$ is an increasing function on $[0, \infty)$, we have for every $\beta \leq \alpha$ and $x \in \overline{\mathbb{R}}^{N}$ that

$$
\left|\partial^{\beta} x^{\alpha}\right| \leq \prod_{i=1}^{N} \alpha_{i} ! e^{-\frac{a}{b}\left(\alpha_{i}-\beta_{i}\right)} e^{\frac{1}{b} \omega\left(\left|x_{i}\right|\right)} \leq \alpha ! e^{-\frac{a}{b}|\alpha|} e^{\frac{|\alpha|}{b} \omega(x)}
$$

Accordingly, $x^{\alpha} \in \mathcal{O}_{M, \omega}\left(\mathbb{R}^{N}\right)$.

(3) The function $\log \left(1+|x|^{2}\right) \in \mathcal{O}_{C, \omega}\left(\mathbb{R}^{N}\right)$ and the proof follows as in the case (2) above.

We now give an useful characterization of the elements of the spaces $\mathcal{O}_{M, \omega}\left(\mathbb{R}^{N}\right)$ and $\mathcal{O}_{C, \omega}\left(\mathbb{R}^{N}\right)$.

Proposition 3.3 Let $\omega$ be a non-quasianalytic weight function. Then the following properties are satified.

(1) A function $f \in C^{\infty}\left(\mathbb{R}^{N}\right)$ is a slowly increasing function of Beurling type if and only if $f \in \mathcal{E}_{\omega}\left(\mathbb{R}^{N}\right)$ and for each $m \in \mathbb{N}$ there exist $C, R>0$ and $n \in \mathbb{N}$ such that for every $\alpha \in \mathbb{N}_{0}^{N}$ and $x \in \mathbb{R}^{N}$ with $|x| \geq R$ we have

$$
\left|\partial^{\alpha} f(x)\right| \leq C \exp \left(n \omega(x)+m \varphi_{\omega}^{*}\left(\frac{|\alpha|}{m}\right)\right) .
$$

(2) A function $f \in C^{\infty}\left(\mathbb{R}^{N}\right)$ is a very slowly increasing function of Beurling type if and only if $f \in \mathcal{E}_{\omega}\left(\mathbb{R}^{N}\right)$ and there exists $n \in \mathbb{N}$ such that for every $m \in \mathbb{N}$ there exist $C, R>0$ so that for every $\alpha \in \mathbb{N}_{0}^{N}$ and $x \in \mathbb{R}^{N}$ with $|x| \geq R$ we have

$$
\left|\partial^{\alpha} f(x)\right| \leq C \exp \left(n \omega(x)+m \varphi_{\omega}^{*}\left(\frac{|\alpha|}{m}\right)\right) .
$$

Proof (1) The necessity of the condition (3.6) is obvious. We need only to prove that $f \in \mathcal{E}_{\omega}\left(\mathbb{R}^{N}\right)$. So, fix a compact subset $K$ of $\mathbb{R}^{N}$. Then for each $m \in \mathbb{N}$ there exists $n \in \mathbb{N}$ such that for every $\alpha \in \mathbb{N}_{0}^{N}$ and $x \in K$ we have

$$
\left|\partial^{\alpha} f(x)\right| \leq r_{m, n}(f) \exp \left(n \omega(x)+m \varphi_{\omega}^{*}\left(\frac{|\alpha|}{m}\right)\right) \leq D r_{m, n}(f) \exp \left(m \varphi_{\omega}^{*}\left(\frac{|\alpha|}{m}\right)\right)
$$

where $D:=\max _{y \in K}\{\exp (n \omega(y))\}<\infty$ is a constant depending only on $n$ and $K$. Hence, it follows for every $m \in \mathbb{N}$ that

$$
p_{K, m}(f)=\sup _{\alpha \in \mathbb{N}_{0}^{N}} \sup _{x \in K}\left|\partial^{\alpha} f(x)\right| \exp \left(-m \varphi_{\omega}^{*}\left(\frac{|\alpha|}{m}\right)\right) \leq D r_{m, n}(f)<\infty .
$$

Since $K$ is arbitrary, this implies that $f \in \mathcal{E}_{\omega}\left(\mathbb{R}^{N}\right)$.

We now prove the sufficiency of the condition. 
Fix $m \in \mathbb{N}$. By assumption there exist $C, R>0$ and $n \in \mathbb{N}$ such that the inequality (3.6) is satisfied and $f \in \mathcal{E}_{\omega}\left(\mathbb{R}^{N}\right)$. Accordingly, we have

$$
K_{1}:=\sup _{\alpha \in \mathbb{N}_{0}^{N}} \sup _{|x| \leq R}\left|\partial^{\alpha} f(x)\right| \exp \left(-m \varphi_{\omega}^{*}\left(\frac{|\alpha|}{m}\right)\right)<\infty .
$$

If we set $K_{2}:=\min _{|x| \leq R} \exp (n \omega(x)) \geq 1$, then we obtain for every $|x| \leq R$ and $\alpha \in \mathbb{N}_{0}^{N}$ that

$$
\begin{aligned}
\left|\partial^{\alpha} f(x)\right| & \leq K_{1} \exp \left(m \varphi_{\omega}^{*}\left(\frac{|\alpha|}{m}\right)\right)=\frac{K_{1}}{K_{2}} K_{2} \exp \left(m \varphi_{\omega}^{*}\left(\frac{|\alpha|}{m}\right)\right) \\
& \leq \frac{K_{1}}{K_{2}} \exp (n \omega(x)) \exp \left(m \varphi_{\omega}^{*}\left(\frac{|\alpha|}{m}\right)\right) .
\end{aligned}
$$

Therefore, setting $C^{\prime}:=\max \left\{\frac{K_{1}}{K_{2}}, C\right\}$, the inequality (3.2) follows.

(2) follows by the same arguments for the proof of property (1) above.

We now establish some features concerning topological properties of the spaces introduced in Definition 3.1.

Proposition 3.4 Let $\omega$ be a non-quasianalytic weight function. Then the following properties are satisfied.

(1) Let $m \in \mathbb{N}$ and $n \in \mathbb{Z}$. Then $\left(\mathcal{O}_{n, \omega}^{m}\left(\mathbb{R}^{N}\right), r_{m, n}\right)$ is a Banach space.

(2) For every $n, n^{\prime} \in \mathbb{Z}$ with $n \leq n^{\prime}$ and $m \in \mathbb{N}$, the inclusion

$$
\left(\mathcal{O}_{n, \omega}^{m}\left(\mathbb{R}^{N}\right), r_{m, n}\right) \hookrightarrow\left(\mathcal{O}_{n^{\prime}, \omega}^{m}\left(\mathbb{R}^{N}\right), r_{m, n^{\prime}}\right)
$$

is well-defined and continuous.

(3) For every $n \in \mathbb{Z}$ and $m, m^{\prime} \in \mathbb{N}$ with $m \leq m^{\prime}$, the inclusion

$$
\left(\mathcal{O}_{n, \omega}^{m^{\prime}}\left(\mathbb{R}^{N}\right), r_{m^{\prime}, n}\right) \hookrightarrow\left(\mathcal{O}_{n, \omega}^{m}\left(\mathbb{R}^{N}\right), r_{m, n}\right)
$$

is well-defined and continuous.

Proof (1) Fix $m \in \mathbb{N}$ and $n \in \mathbb{Z}$. It sufficies to show only the completeness. So, we fix a Cauchy sequence $\left\{f_{j}\right\}_{j \in \mathbb{N}}$ in $\left(\mathcal{O}_{n, \omega}^{m}\left(\mathbb{R}^{N}\right), r_{m, n}\right)$ and observe that

$$
\left|\partial^{\alpha} f_{j}(x)-\partial^{\alpha} f_{j^{\prime}}(x)\right| \leq \exp \left(n \omega(x)+m \varphi_{\omega}^{*}\left(\frac{|\alpha|}{m}\right)\right) r_{m, n}\left(f_{j}-f_{j^{\prime}}\right)
$$

for all $j, j^{\prime} \in \mathbb{N}, \alpha \in \mathbb{N}_{0}^{N}$ and $x \in \mathbb{R}^{N}$. Therefore, for any compact subset $K$ of $\mathbb{R}^{N}$, we have

$$
\sup _{\beta \leq \alpha} \sup _{x \in K}\left|\partial^{\beta} f_{j}(x)-\partial^{\beta} f_{j^{\prime}}(x)\right| \leq C_{K, \alpha} r_{m, n}\left(f_{j}-f_{j^{\prime}}\right),
$$


for all $j, j^{\prime} \in \mathbb{N}$ and $\alpha \in \mathbb{N}_{0}^{N}$, where $C_{K, \alpha}:=\sup _{x \in K} \exp (n \omega(x)) \sup _{\beta \leq \alpha}$ $\exp \left(m \varphi_{\omega}^{*}\left(\frac{|\alpha|}{m}\right)\right)<\infty$. Since $r_{m, n}\left(f_{j}-f_{j^{\prime}}\right) \rightarrow 0$ as $j, j^{\prime} \rightarrow \infty$, and $C^{\infty}\left(\mathbb{R}^{N}\right)$ is a Fréchet space, it follows that $\left\{f_{j}\right\}_{j \in \mathbb{N}}$ is also a Cauchy sequence in $C^{\infty}\left(\mathbb{R}^{N}\right)$. Accordingly, there exists $f \in C^{\infty}\left(\mathbb{R}^{N}\right)$ such that $f_{j} \rightarrow f$ in $C^{\infty}\left(\mathbb{R}^{N}\right)$, as $j \rightarrow \infty$. In particular, $\partial^{\alpha} f_{j} \rightarrow \partial^{\alpha} f$ uniformly on compact subsets of $\mathbb{R}^{N}$ for every $\alpha \in \mathbb{N}_{0}^{N}$.

Now, fix $\epsilon>0$. Since $\left\{f_{j}\right\}_{j \in \mathbb{N}}$ is a Cauchy sequence in $\left(\mathcal{O}_{n, \omega}^{m}\left(\mathbb{R}^{N}\right), r_{m, n}\right)$, there exists $j_{0} \in \mathbb{N}$ such that $r_{m, n}\left(f_{j}-f_{j^{\prime}}\right) \leq \epsilon$ for all $j, j^{\prime} \geq j_{0}$. It follows by (3.10) that for every $j, j^{\prime} \geq j_{0}, x \in \mathbb{R}^{N}$ and $\alpha \in \mathbb{N}_{0}^{N}$ we have

$$
\left|\partial^{\alpha} f_{j}(x)-\partial^{\alpha} f_{j^{\prime}}(x)\right| \leq \epsilon \exp \left(n \omega(x)+m \varphi_{\omega}^{*}\left(\frac{|\alpha|}{m}\right)\right) .
$$

Letting $j^{\prime} \rightarrow \infty$ in the inequality above, we obtain for every $j \geq j_{0}, x \in \mathbb{R}^{N}$ and $\alpha \in \mathbb{N}_{0}^{N}$ that

$$
\left|\partial^{\alpha} f_{j}(x)-\partial^{\alpha} f(x)\right| \leq \epsilon \exp \left(n \omega(x)+m \varphi_{\omega}^{*}\left(\frac{|\alpha|}{m}\right)\right) .
$$

Accordingly, we have

$$
\begin{aligned}
& r_{m, n}\left(f_{j}-f\right) \leq \epsilon \quad \forall j \geq j_{0}, \\
& r_{m, n}(f) \leq r_{m, n}\left(f-f_{j_{0}}\right)+r_{m, n}\left(f_{j_{0}}\right) \leq \epsilon+r_{m, n}\left(f_{j_{0}}\right)<\infty
\end{aligned}
$$

This means that $f \in \mathcal{O}_{n, \omega}^{m}\left(\mathbb{R}^{N}\right)$ and that $f_{j} \rightarrow f$ in $\left(\mathcal{O}_{n, \omega}^{m}\left(\mathbb{R}^{N}\right), r_{m, n}\right)$ for $j \rightarrow \infty$, as $\epsilon>0$ is arbitrary.

(2) Fix $n, n^{\prime} \in \mathbb{Z}$ with $n \leq n^{\prime}$ and $m \in \mathbb{N}$. Then for every $f \in \mathcal{O}_{n, \omega}^{m}\left(\mathbb{R}^{N}\right)$, we have

$$
\begin{aligned}
r_{m, n^{\prime}}(f) & =\sup _{\alpha \in \mathbb{N}_{0}^{N}} \sup _{x \in \mathbb{R}^{N}}\left|\partial^{\alpha} f(x)\right| \exp \left(-n^{\prime} \omega(x)-m \varphi_{\omega}^{*}\left(\frac{|\alpha|}{m}\right)\right) \\
& \leq \sup _{\alpha \in \mathbb{N}_{0}^{N}} \sup _{x \in \mathbb{R}^{N}}\left|\partial^{\alpha} f(x)\right| \exp \left(-n \omega(x)-m \varphi_{\omega}^{*}\left(\frac{|\alpha|}{m}\right)\right)=r_{m, n}(f) .
\end{aligned}
$$

Therefore, the inclusion in (3.8) is well-defined and continuous.

(3) Fix $m, m^{\prime} \in \mathbb{N}$ with $m \leq m^{\prime}$ and $n \in \mathbb{Z}$. Since $\varphi_{\omega}^{*}(t) / t$ is an increasing function in $(0, \infty)$ (see Lemma $2.3(2))$, for every $f \in \mathcal{O}_{n, \omega}^{m^{\prime}}\left(\mathbb{R}^{N}\right)$ we have

$$
\begin{aligned}
r_{m, n}(f) & =\sup _{\alpha \in \mathbb{N}_{0}^{N}} \sup _{x \in \mathbb{R}^{N}}\left|\partial^{\alpha} f(x)\right| \exp \left(-n \omega(x)-m \varphi_{\omega}^{*}\left(\frac{|\alpha|}{m}\right)\right) \\
& \leq \sup _{\alpha \in \mathbb{N}_{0}^{N}} \sup _{x \in \mathbb{R}^{N}}\left|\partial^{\alpha} f(x)\right| \exp \left(-n \omega(x)-m^{\prime} \varphi_{\omega}^{*}\left(\frac{|\alpha|}{m^{\prime}}\right)\right)=r_{m^{\prime}, n}(f) .
\end{aligned}
$$

Therefore, the inclusion in (3.9) is well-defined and continuous. 
Via Proposition 3.4(1)-(2) we deduce that the sequence $\left\{\left(\mathcal{O}_{n, \omega}^{m}\left(\mathbb{R}^{N}\right), r_{m, n}\right)\right\}_{n \in \mathbb{N}}$ of Banach spaces forms for each $m \in \mathbb{N}$ an inductive spectrum. The space $\bigcup_{n=1}^{\infty} \mathcal{O}_{n, \omega}^{m}\left(\mathbb{R}^{N}\right)$, endowed with the inductive topology defined by $\left\{\left(\mathcal{O}_{n, \omega}^{m}\left(\mathbb{R}^{N}\right), r_{m, n}\right)\right\}_{n \in \mathbb{N}}$, is then an (LB)-space for each $m \in \mathbb{N}$. On the other hand, via Proposition 3.4(1)-(3) we also deduce for every $n \in \mathbb{N}$ that the sequence $\left\{\left(\mathcal{O}_{n, \omega}^{m}\left(\mathbb{R}^{N}\right), r_{m, n}\right)\right\}_{m \in \mathbb{N}}$ of Banach spaces forms a projective spectrum. So, for every $n \in \mathbb{N}$ the space $\bigcap_{m=1}^{\infty} \mathcal{O}_{n, \omega}^{m}\left(\mathbb{R}^{N}\right)$, endowed with the projective topology defined by $\left\{\left(\mathcal{O}_{n, \omega}^{m}\left(\mathbb{R}^{N}\right), r_{m, n}\right)\right\}_{m \in \mathbb{N}}$, is a Fréchet space. In the following we always suppose that the spaces $\bigcup_{n=1}^{\infty} \mathcal{O}_{n, \omega}^{m}\left(\mathbb{R}^{N}\right)$ and $\bigcap_{m=1}^{\infty} \mathcal{O}_{n, \omega}^{m}\left(\mathbb{R}^{N}\right)$ are equipped respectively with the (LB)-topology and Fréchet topology defined above. In particular, the spaces $\bigcup_{n=1}^{\infty} \mathcal{O}_{n, \omega}^{m}\left(\mathbb{R}^{N}\right)$ and $\bigcap_{m=1}^{\infty} \mathcal{O}_{n, \omega}^{m}\left(\mathbb{R}^{N}\right)$ satisfy the following properties.

Proposition 3.5 Let $\omega$ be a non-quasianalytic weight function and $m \in \mathbb{N}$. Then $\bigcup_{n=1}^{\infty} \mathcal{O}_{n, \omega}^{m}\left(\mathbb{R}^{N}\right)$ is a complete (LB)-space.

Proof In order to show the completeness of the space $\bigcup_{n=1}^{\infty} \mathcal{O}_{n, \omega}^{m}\left(\mathbb{R}^{N}\right)$, we first prove that the inclusion $\bigcup_{n=1}^{\infty} \mathcal{O}_{n, \omega}^{m}\left(\mathbb{R}^{N}\right) \hookrightarrow C^{\infty}\left(\mathbb{R}^{N}\right)$ is continuous as follows.

Fix $n \in \mathbb{N}$. Then for every compact subset $K$ of $\mathbb{R}^{N}$ and $\alpha \in \mathbb{N}_{0}^{N}$ we have

$$
\begin{aligned}
& \sup _{x \in K} \sup _{\beta \leq \alpha}\left|\partial^{\beta} f(x)\right| \\
& \quad=\sup _{x \in K} \sup _{\beta \leq \alpha}\left|\partial^{\beta} f(x)\right| \exp \left(-n \omega(x)-m \varphi_{\omega}^{*}\left(\frac{|\beta|}{m}\right)\right) \exp \left(n \omega(x)+m \varphi_{\omega}^{*}\left(\frac{|\beta|}{m}\right)\right) \\
& \leq C_{K, \alpha} r_{m, n}(f)
\end{aligned}
$$

for each $f \in \mathcal{O}_{n, \omega}^{m}\left(\mathbb{R}^{N}\right)$, where $C_{K, \alpha}:=\sup _{x \in K} \sup _{\beta \leq \alpha} \exp \left(n \omega(x)+m \varphi_{\omega}^{*}\left(\frac{|\beta|}{m}\right)\right)<$ $\infty$ is a positive constant depending on $K$ and $\alpha$. This means that the inclusion $\left(\mathcal{O}_{n, \omega}^{m}\left(\mathbb{R}^{N}\right), r_{m, n}\right) \hookrightarrow C^{\infty}\left(\mathbb{R}^{N}\right)$ is continuous. Since $n \in \mathbb{N}$ is arbitrary and $\bigcup_{n=1}^{\infty} \mathcal{O}_{n, \omega}^{m}\left(\mathbb{R}^{N}\right)$ is an (LB)-space, the inclusion $\bigcup_{n=1}^{\infty} \mathcal{O}_{n, \omega}^{m}\left(\mathbb{R}^{N}\right) \hookrightarrow C^{\infty}\left(\mathbb{R}^{N}\right)$ is continuous too. Therefore, there exists a Hausdorff lc-topology $\tau$ on $\bigcup_{n=1}^{\infty} \mathcal{O}_{n, \omega}^{m}\left(\mathbb{R}^{N}\right)$ with the property that the closed unit ball of each $\mathcal{O}_{n, \omega}^{m}\left(\mathbb{R}^{N}\right)$ is relatively $\tau$-compact. But, the closed unit ball of each $\mathcal{O}_{n, \omega}^{m}\left(\mathbb{R}^{N}\right)$ is also $\tau$-compact. Indeed, let $n \in \mathbb{N}$ and let $\left\{f_{j}\right\}_{j \in N} \subseteq\left\{g \in \mathcal{O}_{n, \omega}^{m}\left(\mathbb{R}^{N}\right): r_{m, n}(g) \leq 1\right\} \tau$-convergent to some $f \in C^{\infty}\left(\mathbb{R}^{N}\right)$. Then for every $x \in \mathbb{R}^{N}, j \in \mathbb{N}$ and $\alpha \in \mathbb{N}_{0}^{N}$ we have

$$
\left|\partial^{\alpha} f_{j}(x)\right| \leq \exp \left(n \omega(x)+m \varphi_{\omega}^{*}\left(\frac{|\alpha|}{m}\right)\right) .
$$

Since $f_{j} \rightarrow f$ in $C^{\infty}\left(\mathbb{R}^{N}\right)$ as $j \rightarrow \infty$ and hence $\partial^{\alpha} f_{j} \rightarrow \partial^{\alpha} f$ pointwise on $\mathbb{R}^{N}$ for each $\alpha \in \mathbb{N}_{0}^{N}$, it follows by letting $j \rightarrow \infty$ in (3.11) for every $x \in \mathbb{R}^{N}$ and $\alpha \in \mathbb{N}_{0}^{N}$ that

$$
\left|\partial^{\alpha} f(x)\right| \leq \exp \left(n \omega(x)+m \varphi_{\omega}^{*}\left(\frac{|\alpha|}{m}\right)\right) .
$$


This implies that $r_{m, n}(f) \leq 1$. So, $\left\{g \in \mathcal{O}_{n, \omega}^{m}\left(\mathbb{R}^{N}\right): r_{m, n}(g) \leq 1\right\}$ is $\tau$-closed.

The result now follows by Mujica [23, Theorem 1].

Proposition 3.6 Let $\omega$ be a non-quasianalytic weight function. Then the following properties are satisfied.

(1) For every $n, n^{\prime} \in \mathbb{Z}$ with $n \leq n^{\prime}$, the inclusion

$$
\bigcap_{m=1}^{\infty} \mathcal{O}_{n, \omega}^{m}\left(\mathbb{R}^{N}\right) \hookrightarrow \bigcap_{m=1}^{\infty} \mathcal{O}_{n^{\prime}, \omega}^{m}\left(\mathbb{R}^{N}\right)
$$

is well-defined and continuous.

(2) For every $m, m^{\prime} \in \mathbb{N}$ with $m \leq m^{\prime}$, the inclusion

$$
\bigcup_{n=1}^{\infty} \mathcal{O}_{n, \omega}^{m^{\prime}}\left(\mathbb{R}^{N}\right) \hookrightarrow \bigcup_{n=1}^{\infty} \mathcal{O}_{n, \omega}^{m}\left(\mathbb{R}^{N}\right)
$$

is well-defined and continuous.

Proof (1) Fix $n, n^{\prime} \in \mathbb{Z}$ with $n \leq n^{\prime}$. Then, for every $h \in \mathbb{N}$ and $f \in \bigcap_{m=1}^{\infty} \mathcal{O}_{n, \omega}^{m}\left(\mathbb{R}^{N}\right)$, we have

$$
\begin{aligned}
r_{h, n^{\prime}}(f) & =\sup _{\alpha \in \mathbb{N}_{0}^{N}} \sup _{x \in \mathbb{R}^{N}}\left|\partial^{\alpha} f(x)\right| \exp \left(-n^{\prime} \omega(x)-h \varphi_{\omega}^{*}\left(\frac{|\alpha|}{h}\right)\right) \\
& \leq \sup _{\alpha \in \mathbb{N}_{0}^{N}} \sup _{x \in \mathbb{R}^{N}}\left|\partial^{\alpha} f(x)\right| \exp \left(-n \omega(x)-h \varphi_{\omega}^{*}\left(\frac{|\alpha|}{h}\right)\right)=r_{h, n}(f) .
\end{aligned}
$$

This means that the inclusion in (3.12) is well-defined and continuous.

(2) Fix $m, m^{\prime} \in \mathbb{N}$ with $m \leq m^{\prime}$ and $h \in \mathbb{N}$. Then by Proposition 3.4(3) the inclusion $\left(\mathcal{O}_{h, \omega}^{m^{\prime}}\left(\mathbb{R}^{N}\right), r_{m^{\prime}, h}\right) \hookrightarrow\left(\mathcal{O}_{h, \omega}^{m}\left(\mathbb{R}^{N}\right), r_{m, h}\right)$ is continuous. On the other hand, the inclusion $\left(\mathcal{O}_{h, \omega}^{m}\left(\mathbb{R}^{N}\right), r_{m, h}\right) \hookrightarrow \bigcup_{n=1}^{\infty} \mathcal{O}_{n, \omega}^{m}\left(\mathbb{R}^{N}\right)$ is also continuous. Accordingly, the inclusion

$$
\left(\mathcal{O}_{h, \omega}^{m^{\prime}}\left(\mathbb{R}^{N}\right), r_{m^{\prime}, h}\right) \hookrightarrow \bigcup_{n=1}^{\infty} \mathcal{O}_{n, \omega}^{m}\left(\mathbb{R}^{N}\right)
$$

is continuous. Since $h \in \mathbb{N}$ is arbitrary and $\bigcup_{h=1}^{\infty} \mathcal{O}_{h, \omega}^{m^{\prime}}\left(\mathbb{R}^{N}\right)$ is an (LB)-space, it follows that the inclusion

$$
\bigcup_{h=1}^{\infty} \mathcal{O}_{h, \omega}^{m^{\prime}}\left(\mathbb{R}^{N}\right) \hookrightarrow \bigcup_{n=1}^{\infty} \mathcal{O}_{n, \omega}^{m}\left(\mathbb{R}^{N}\right)
$$

is continuous, i.e., the inclusion in (3.12) is well-defined and continuous. 
Proposition 3.6(1) yields that the sequence $\left\{\bigcap_{m=1}^{\infty} \mathcal{O}_{n, \omega}^{m}\left(\mathbb{R}^{N}\right)\right\}_{n \in \mathbb{N}}$ of Fréchet spaces forms an inductive spectrum. So, we can endow the space $\mathcal{O}_{C, \omega}\left(\mathbb{R}^{N}\right)$ with the inductive topology defined by $\left\{\bigcap_{m=1}^{\infty} \mathcal{O}_{n, \omega}^{m}\left(\mathbb{R}^{N}\right)\right\}_{n \in \mathbb{N}}$. Thereby, $\mathcal{O}_{C, \omega}\left(\mathbb{R}^{N}\right)$, equipped with such an inductive topology, is an (LF)-space. Proposition 3.6(2) also yields that the sequence $\left\{\bigcup_{n=1}^{\infty} \mathcal{O}_{n, \omega}^{m}\left(\mathbb{R}^{N}\right)\right\}_{m \in \mathbb{N}}$ of (LB)-spaces is a projective spectrum. So, we can endow the space $\mathcal{O}_{M, \omega}\left(\mathbb{R}^{N}\right)$ with the projective topology defined by $\left\{\bigcup_{n=1}^{\infty} \mathcal{O}_{n, \omega}^{m}\left(\mathbb{R}^{N}\right)\right\}_{m \in \mathbb{N}}$. Thereby, $\mathcal{O}_{M, \omega}\left(\mathbb{R}^{N}\right)$, equipped with this projective topology, is a projective limit of (LB)-spaces. By Proposition 3.5, it is then a complete lc-space. In the following we always assume that the spaces $\mathcal{O}_{C, \omega}\left(\mathbb{R}^{N}\right)$ and $\mathcal{O}_{M, \omega}\left(\mathbb{R}^{N}\right)$ are endowed with the lc-topologies defined above.

Remark 3.7 We observe that, for fixed $m \in \mathbb{N}$ and $n \in \mathbb{N}$, we have that

$$
q_{m, n}(f)=\sup _{\alpha \in \mathbb{N}_{0}^{N}} \sup _{x \in \mathbb{R}^{N}} \exp \left(-m \varphi_{\omega}^{*}\left(\frac{|\alpha|}{m}\right)\right) \exp (n \omega(x))\left|\partial^{\alpha} f(x)\right|=r_{m,-n}(f) .
$$

for every $f \in \mathcal{S}_{\omega}\left(\mathbb{R}^{N}\right)$. Since $\left\{q_{m, n}\right\}_{m, n \in \mathbb{N}}$ is a fundamental sequence of norms generating the Fréchet topology of $\mathcal{S}_{\omega}\left(\mathbb{R}^{N}\right)$, it follows that $\mathcal{S}_{\omega}\left(\mathbb{R}^{N}\right)=\bigcap_{n=1}^{\infty} \bigcap_{m=1}^{\infty} \mathcal{O}_{-n, \omega}^{m}$ $\left(\mathbb{R}^{N}\right)$.

Theorem 3.8 Let $\omega$ be a non-quasianalytic weight function. Then the following properties are satisfied.

(1) The inclusion

$$
\mathcal{O}_{C, \omega}\left(\mathbb{R}^{N}\right) \hookrightarrow \mathcal{O}_{M, \omega}\left(\mathbb{R}^{N}\right)
$$

is well-defined and continuous.

(2) The inclusions

$$
\mathcal{S}_{\omega}\left(\mathbb{R}^{N}\right) \hookrightarrow \mathcal{O}_{C, \omega}\left(\mathbb{R}^{N}\right) \hookrightarrow \mathcal{E}_{\omega}\left(\mathbb{R}^{N}\right)
$$

are well-defined and continuous.

Proof (1) Fix $f \in \mathcal{O}_{C, \omega}\left(\mathbb{R}^{N}\right)$ and $m \in \mathbb{N}$. Then there exists $n \in \mathbb{N}$ such that $r_{m, n}(f)<$ $\infty$ and so, $f \in \mathcal{O}_{n, \omega}^{m}\left(\mathbb{R}^{N}\right) \subset \bigcup_{h=1}^{\infty} \mathcal{O}_{h, \omega}^{m}\left(\mathbb{R}^{N}\right)$. Since $m \in \mathbb{N}$ is arbitrary, we can conclude that $f \in \mathcal{O}_{M, \omega}\left(\mathbb{R}^{N}\right)$. We have so shown that the inclusion is well-defined. We now prove that such an inclusion is continuous as follows.

We first observe that for every $m^{\prime} \in \mathbb{N}$ and $n, n^{\prime} \in \mathbb{N}$ with $n \leq n^{\prime}$, the inclusion

$$
\bigcap_{m=1}^{\infty} \mathcal{O}_{n, \omega}^{m}\left(\mathbb{R}^{N}\right) \hookrightarrow \mathcal{O}_{n^{\prime}, \omega}^{m^{\prime}}\left(\mathbb{R}^{N}\right)
$$

is well-defined and continuous. Indeed, for every $f \in \bigcap_{m=1}^{\infty} \mathcal{O}_{n, \omega}^{m}\left(\mathbb{R}^{N}\right)$ we have

$$
r_{m^{\prime}, n^{\prime}}(f)=\sup _{\alpha \in \mathbb{N}_{0}^{N}} \sup _{x \in \mathbb{R}^{N}}\left|\partial^{\alpha} f(x)\right| \exp \left(-n^{\prime} \omega(x)-m^{\prime} \varphi_{\omega}^{*}\left(\frac{|\alpha|}{m^{\prime}}\right)\right)
$$




$$
\leq \sup _{\alpha \in \mathbb{N}_{0}^{N}} \sup _{x \in \mathbb{R}^{N}}\left|\partial^{\alpha} f(x)\right| \exp \left(-n \omega(x)-m^{\prime} \varphi_{\omega}^{*}\left(\frac{|\alpha|}{m^{\prime}}\right)\right)=r_{m^{\prime}, n}(f) .
$$

Since $r_{m^{\prime}, n} \in\left\{r_{m, n}\right\}_{m \in \mathbb{N}}$ and $\left\{r_{m, n}\right\}_{m \in \mathbb{N}}$ is a sequence of norms defining the lc-topology of $\bigcap_{m=1}^{\infty} \mathcal{O}_{n, \omega}^{m}\left(\mathbb{R}^{N}\right)$, it follows that the inclusion $\bigcap_{m=1}^{\infty} \mathcal{O}_{n, \omega}^{m}\left(\mathbb{R}^{N}\right) \hookrightarrow \mathcal{O}_{n^{\prime}, \omega}^{m^{\prime}}\left(\mathbb{R}^{N}\right)$ is continuous. Taking into account that $\bigcup_{h=1}^{\infty} \mathcal{O}_{h, \omega}^{m^{\prime}}\left(\mathbb{R}^{N}\right)$ is an (LB)-space and so, $\mathcal{O}_{n^{\prime}, \omega}^{m^{\prime}}\left(\mathbb{R}^{N}\right) \hookrightarrow \bigcup_{h=1}^{\infty} \mathcal{O}_{h, \omega}^{m^{\prime}}\left(\mathbb{R}^{N}\right)$ continuously, we deduce for every $m^{\prime} \in \mathbb{N}$ and $n \in \mathbb{N}$ that the inclusion

$$
\bigcap_{m=1}^{\infty} \mathcal{O}_{n, \omega}^{m}\left(\mathbb{R}^{N}\right) \hookrightarrow \bigcup_{h=1}^{\infty} \mathcal{O}_{h, \omega}^{m^{\prime}}\left(\mathbb{R}^{N}\right)
$$

is well-defined and continuous. Since $\mathcal{O}_{M, \omega}\left(\mathbb{R}^{N}\right)=\bigcap_{m^{\prime}=1}^{\infty} \bigcup_{h=1}^{\infty} \mathcal{O}_{h, \omega}^{m^{\prime}}\left(\mathbb{R}^{N}\right)$ is the projective limit of the sequence $\left\{\bigcup_{h=1}^{\infty} \mathcal{O}_{h, \omega}^{m^{\prime}}\left(\mathbb{R}^{N}\right)\right\}_{m^{\prime} \in \mathbb{N}}$ of (LB)-spaces, it follows for every $n \in \mathbb{N}$ that the inclusion

$$
\bigcap_{m=1}^{\infty} \mathcal{O}_{n, \omega}^{m}\left(\mathbb{R}^{N}\right) \hookrightarrow \mathcal{O}_{M, \omega}\left(\mathbb{R}^{N}\right)
$$

is well-defined and continuous. Finally, since $\mathcal{O}_{C, \omega}\left(\mathbb{R}^{N}\right)$ is the inductive limit of the sequence $\left\{\bigcap_{m=1}^{\infty} \mathcal{O}_{n, \omega}^{m}\left(\mathbb{R}^{N}\right)\right\}_{n \in \mathbb{N}}$ of Fréchet space, it follows that the inclusion

$$
\mathcal{O}_{C, \omega}\left(\mathbb{R}^{N}\right) \hookrightarrow \mathcal{O}_{M, \omega}\left(\mathbb{R}^{N}\right)
$$

is continuous.

(2) We first show that the inclusion $\mathcal{O}_{C, \omega}\left(\mathbb{R}^{N}\right) \hookrightarrow \mathcal{E}_{\omega}\left(\mathbb{R}^{N}\right)$ is continuous. So, fix $f \in \mathcal{O}_{C, \omega}\left(\mathbb{R}^{N}\right)$ and $n \in \mathbb{N}$. Then $f \in \bigcap_{h=1}^{\infty} \mathcal{O}_{n, \omega}^{h}\left(\mathbb{R}^{N}\right)$. This implies for every $m \in \mathbb{N}$ and $K$ compact subset of $\mathbb{R}^{N}$ that

$$
\begin{aligned}
p_{K, m}(f) & =\sup _{\alpha \in \mathbb{N}_{0}^{N}} \sup _{x \in K}\left|\partial^{\alpha} f(x)\right| \exp \left(-m \varphi_{\omega}^{*}\left(\frac{|\alpha|}{m}\right)\right) \\
& =\sup _{\alpha \in \mathbb{N}_{0}^{N}} \sup _{x \in K}\left|\partial^{\alpha} f(x)\right| \exp \left(-m \varphi_{\omega}^{*}\left(\frac{|\alpha|}{m}\right)\right) \exp (n \omega(x)-n \omega(x)) \\
& \leq D \sup _{\alpha \in \mathbb{N}_{0}^{N}} \sup _{x \in K}\left|\partial^{\alpha} f(x)\right| \exp \left(-m \varphi_{\omega}^{*}\left(\frac{|\alpha|}{m}\right)\right) \exp (-n \omega(x)) \leq D r_{m, n}(f)<\infty,
\end{aligned}
$$

where $D:=\max _{y \in K}\{\exp (n \omega(y))\}<\infty$ is a constant depending only on $n$ and $K$. Since $\left\{r_{m, n}\right\}_{m \in \mathbb{N}}$ is a sequence of norms generating the lc-topology of the Fréchet space $\bigcap_{h=1}^{\infty} \mathcal{O}_{n, \omega}^{h}\left(\mathbb{R}^{N}\right)$ and $\left\{p_{K, m}\right\}_{K \Subset \mathbb{R}^{N}}$ is a sequence of seminorms generating the lc-topology of the Fréchet space $\mathcal{E}_{\omega}\left(\mathbb{R}^{N}\right)$, it follows that the inclusion 


$$
\bigcap_{h=1}^{\infty} \mathcal{O}_{n, \omega}^{h}\left(\mathbb{R}^{N}\right) \hookrightarrow \mathcal{E}_{\omega}\left(\mathbb{R}^{N}\right)
$$

is continuous. Since $n \in \mathbb{N}$ is arbitrary and $\mathcal{O}_{C, \omega}\left(\mathbb{R}^{N}\right)$ is the inductive limit of the Fréchet spaces $\left\{\bigcap_{h=1}^{\infty} \mathcal{O}_{n, \omega}^{h}\left(\mathbb{R}^{N}\right)\right\}_{n \in \mathbb{N}}$, it follows that the inclusion on the right of (3.15) is continuous.

We now show that the inclusion $\mathcal{S}_{\omega}\left(\mathbb{R}^{N}\right) \hookrightarrow \mathcal{O}_{C, \omega}\left(\mathbb{R}^{N}\right)$ is continuous. So, fix $f \in \mathcal{S}_{\omega}\left(\mathbb{R}^{N}\right)$. Then for every $m, n \in \mathbb{N}$ we have that $q_{m, n}(f)<\infty$, thereby implying that

$$
\begin{aligned}
r_{m, n}(f) & =\alpha \in \mathbb{N}_{0}^{N} \sup \sup _{x \in \mathbb{R}^{N}} \exp \left(-m \varphi_{\omega}^{*}\left(\frac{|\alpha|}{m}\right)\right) \exp (-n \omega(x))\left|\partial^{\alpha} f(x)\right| \\
& <q_{m, n}(f)<\infty
\end{aligned}
$$

Hence, $f \in \mathcal{O}_{C, \omega}\left(\mathbb{R}^{N}\right)$. This shows that the inclusion is well-defined. To prove the continuity of the inclusion, we observe that for every $n, n^{\prime} \in \mathbb{N}$ and $m \in \mathbb{N}$ the inclusion

$$
\bigcap_{m=1}^{\infty} \mathcal{O}_{-n, \omega}^{m}\left(\mathbb{R}^{N}\right) \hookrightarrow \bigcap_{m=1}^{\infty} \mathcal{O}_{n^{\prime}, \omega}^{m}\left(\mathbb{R}^{N}\right)
$$

is continuous. Since $\bigcap_{m=1}^{\infty} \mathcal{O}_{n^{\prime}, \omega}^{m}\left(\mathbb{R}^{N}\right) \hookrightarrow \mathcal{O}_{C, \omega}\left(\mathbb{R}^{N}\right)$ continuously for all $n^{\prime} \in \mathbb{N}$, it follows for every $m \in \mathbb{N}$ that the inclusion

$$
\bigcap_{m=1}^{\infty} \mathcal{O}_{-n, \omega}^{m}\left(\mathbb{R}^{N}\right) \hookrightarrow \mathcal{O}_{C, \omega}\left(\mathbb{R}^{N}\right)
$$

is continuous. Accordingly, as $\mathcal{S}_{\omega}\left(\mathbb{R}^{N}\right)$ is the projective limit of the Fréchet spaces $\left\{\bigcap_{m=1}^{\infty} \mathcal{O}_{-n, \omega}^{m}\left(\mathbb{R}^{N}\right)\right\}_{n \in \mathbb{N}}$, we can conclude that the inclusion on the left of (3.14) is continuous.

Finally, $\mathcal{O}_{M, \omega}\left(\mathbb{R}^{N}\right)$ and $\mathcal{O}_{C, \omega}\left(\mathbb{R}^{N}\right)$ have the following important property.

Theorem 3.9 Let $\omega$ be a non-quasianalytic weight function. Then the following inclusions

$$
\mathcal{D}_{\omega}\left(\mathbb{R}^{N}\right) \subseteq \mathcal{S}_{\omega}\left(\mathbb{R}^{N}\right) \subseteq \mathcal{O}_{C, \omega}\left(\mathbb{R}^{N}\right) \subseteq \mathcal{O}_{M, \omega}\left(\mathbb{R}^{N}\right) \subseteq \mathcal{E}_{\omega}\left(\mathbb{R}^{N}\right)
$$

are dense.

Proof The space $\mathcal{D}_{\omega}\left(\mathbb{R}^{N}\right)$ is a dense subspace of $\mathcal{S}_{\omega}\left(\mathbb{R}^{N}\right)$ and of $\mathcal{E}_{\omega}\left(\mathbb{R}^{N}\right)$, see [8, Proposition 4.7.(1)] and [2, Propositions 1.8.6 and 1.8.7]. So, both the spaces $\mathcal{O}_{C, \omega}\left(\mathbb{R}^{N}\right)$ and $\mathcal{O}_{M, \omega}\left(\mathbb{R}^{N}\right)$ are dense subspaces of $\mathcal{E}_{\omega}\left(\mathbb{R}^{N}\right)$.

We now prove that $\mathcal{S}_{\omega}\left(\mathbb{R}^{N}\right)$ is a dense subspace of $\mathcal{O}_{C, \omega}\left(\mathbb{R}^{N}\right)$ as follows. 
Since $\mathcal{D}_{\omega}\left(\mathbb{R}^{N}\right) \subseteq \mathcal{S}_{\omega}\left(\mathbb{R}^{N}\right)$, it suffices to show only that $\mathcal{D}_{\omega}\left(\mathbb{R}^{N}\right)$ is a dense subspace of $\mathcal{O}_{C, \omega}\left(\mathbb{R}^{N}\right)$. So, fix $f \in \mathcal{O}_{C, \omega}\left(\mathbb{R}^{N}\right)$ and $\phi \in \mathcal{D}_{\omega}\left(\mathbb{R}^{N}\right)$ such that $\phi \equiv 1$ on $\bar{B}_{1}(0)$ and $0 \leq \phi \leq 1$. Then for every $\epsilon>0$ the function $\phi_{\epsilon}(x):=\phi(\epsilon x) f(x)$, for $x \in \mathbb{R}^{N}$, belongs to $\overline{\mathcal{D}}_{\omega}\left(\mathbb{R}^{N}\right)$ because $\mathcal{O}_{C, \omega}\left(\mathbb{R}^{N}\right) \subseteq \mathcal{E}_{\omega}\left(\mathbb{R}^{N}\right)$. Since $f \in \mathcal{O}_{C, \omega}\left(\mathbb{R}^{N}\right)$, we have that $f \in \bigcap_{m=1}^{\infty} \mathcal{O}_{n, \omega}^{m}\left(\mathbb{R}^{N}\right)$ for some $n \in \mathbb{N}$. This implies that $f \in \bigcap_{m=1}^{\infty} \mathcal{O}_{h, \omega}^{m}\left(\mathbb{R}^{N}\right)$ for every $h \in \mathbb{N}$ with $h \geq n$. We claim that $r_{m, n+1}\left(\phi_{\epsilon}-f\right) \rightarrow 0$ as $\epsilon \rightarrow 0^{+}$for every $m \in \mathbb{N}$. To show the claim, we proceed as follows.

Fix $m \in \mathbb{N}$ and let $M \in \mathbb{N}$ such that $M \geq L m$, where $L \geq 1$ is the costant appearing in formula (2.2). Then for every $\alpha \in \mathbb{N}_{0}^{N}, x \in \mathbb{R}^{N}$ and $\epsilon>0$ we have

$$
\begin{aligned}
\left|\partial^{\alpha} \phi_{\epsilon}(x)-\partial^{\alpha} f(x)\right| \leq & \left|\partial^{\alpha} f(x)(\phi(\epsilon x)-1)\right|+\sum_{\beta<\alpha}\left(\begin{array}{c}
\alpha \\
\beta
\end{array}\right)\left|\partial^{\beta} f(x)\right| \epsilon^{\alpha-\beta}\left|\partial^{\alpha-\beta} \phi(\epsilon x)\right| \\
\leq & \left|\partial^{\alpha} f(x)(\phi(\epsilon x)-1)\right|+\sum_{\beta<\alpha}\left(\begin{array}{c}
\alpha \\
\beta
\end{array}\right) \epsilon^{\alpha-\beta} r_{M, n+1}(f) \exp ((n+1) \omega(x)) \\
& \times \exp \left(M \varphi_{\omega}^{*}\left(\frac{|\beta|}{M}\right)\right) r_{M, 0}(\phi) \exp \left(M \varphi_{\omega}^{*}\left(\frac{|\alpha-\beta|}{M}\right)\right) \\
\leq & \left|\partial^{\alpha} f(x)(\phi(\epsilon x)-1)\right|+\sum_{\beta<\alpha}\left(\begin{array}{c}
\alpha \\
\beta
\end{array}\right) \epsilon^{\alpha-\beta} r_{M, n+1}(f) \exp ((n+1) \omega(x)) \\
& \times r_{M, 0}(\phi) \exp \left(M \varphi_{\omega}^{*}\left(\frac{|\alpha|}{M}\right)\right) \\
\leq & \left|\partial^{\alpha} f(x)(\phi(\epsilon x)-1)\right| \\
& +r_{M, n+1}(f) r_{M, 0}(\phi) \exp ((n+1) \omega(x)) \exp \left(M \varphi_{\omega}^{*}\left(\frac{|\alpha|}{M}\right)\right) \epsilon 2^{|\alpha|},
\end{aligned}
$$

after having observed that $\sum_{\beta<\alpha}\left(\begin{array}{l}\alpha \\ \beta\end{array}\right) \epsilon^{\alpha-\beta} \leq \epsilon 2^{|\alpha|}$ for every $\alpha \in \mathbb{N}_{0}^{N}$ and using formula (2.7). Since $M \geq m L$, applying the inequality (2.6) it follows via (3.17) for every $\alpha \in \mathbb{N}_{0}^{N}, x \in \mathbb{R}^{N}$ and $\epsilon>0$ that

$$
\begin{aligned}
\left|\partial^{\alpha} \phi_{\epsilon}(x)-\partial^{\alpha} f(x)\right| \leq & \left|\partial^{\alpha} f(x)(\phi(\epsilon x)-1)\right| \\
& +\epsilon C r_{M, n+1}(f) r_{M, 0}(\phi) \exp ((n+1) \omega(x)) \exp \left(m \varphi_{\omega}^{*}\left(\frac{|\alpha|}{m}\right)\right) .
\end{aligned}
$$

Therefore, we have for every $\epsilon>0$ that

$$
\begin{aligned}
r_{m, n+1}\left(\phi_{\epsilon}-f\right)= & \sup _{\alpha \in \mathbb{N}_{0}^{N}} \sup _{x \in \mathbb{R}^{N}} \exp \left(-m \varphi_{\omega}^{*}\left(\frac{|\alpha|}{m}\right)\right) \exp (-(n+1) \omega(x))\left|\partial^{\alpha} \phi_{\epsilon}(x)-\partial^{\alpha} f(x)\right| \\
\leq & \sup _{\alpha \in \mathbb{N}_{0}^{N}} \sup _{x \in \mathbb{R}^{N}} \exp \left(-m \varphi_{\omega}^{*}\left(\frac{|\alpha|}{m}\right)\right) \exp (-(n+1) \omega(x))\left|\partial^{\alpha} f(x)(\phi(\epsilon x)-1)\right| \\
& +\epsilon C r_{M, n+1}(f) r_{M, 0}(\phi) .
\end{aligned}
$$


Since $f \in \bigcap_{m=1}^{\infty} \mathcal{O}_{n, \omega}^{m}\left(\mathbb{R}^{N}\right)$ and $\phi \in \mathcal{D}_{\omega}\left(\mathbb{R}^{N}\right), r_{M, n+1}(f) r_{M, 0}(\phi)<\infty$ and so, we have $\epsilon C r_{M, n+1}(f) r_{M, 0}(\phi) \rightarrow 0$ as $\epsilon \rightarrow 0^{+}$. In order to conclude the proof, it then remains to prove that

$$
\sup _{\alpha \in \mathbb{N}_{0}^{N}} \sup _{x \in \mathbb{R}^{N}} \exp \left(-m \varphi_{\omega}^{*}\left(\frac{|\alpha|}{m}\right)\right) \exp (-(n+1) \omega(x))\left|\partial^{\alpha} f(x)(\phi(\epsilon x)-1)\right| \rightarrow 0, \text { as } \epsilon \rightarrow 0^{+} \text {. }
$$

But, $\phi(\epsilon x)-1=0$ whenever $|x| \leq \frac{1}{\epsilon}$. Accordingly, we have

$$
\begin{aligned}
& \sup _{\alpha \in \mathbb{N}_{0}^{N}} \sup _{x \in \mathbb{R}^{N}} \exp \left(-m \varphi_{\omega}^{*}\left(\frac{|\alpha|}{m}\right)\right) \exp (-(n+1) \omega(x))\left|\partial^{\alpha} f(x)(\phi(\epsilon x)-1)\right| \\
& =\sup _{\alpha \in \mathbb{N}_{0}^{N}} \sup _{|x|>\frac{1}{\epsilon}} \exp \left(-m \varphi_{\omega}^{*}\left(\frac{|\alpha|}{m}\right)\right) \exp (-(n+1) \omega(x))\left|\partial^{\alpha} f(x)(\phi(\epsilon x)-1)\right| \\
& \leq r_{m, n}(f) \sup _{|x|>\frac{1}{\epsilon}} \exp (-\omega(x)) .
\end{aligned}
$$

Since $\sup _{|x|>\frac{1}{\epsilon}} \exp (-\omega(x)) \rightarrow 0$ as $\epsilon \rightarrow 0^{+}$, the claim is proved.

From the arbitarity of $m \in \mathbb{N}$, we can conclude that $\phi_{\epsilon} \rightarrow f$ in $\bigcap_{m=1}^{\infty} \mathcal{O}_{n+1, \omega}^{m}\left(\mathbb{R}^{N}\right)$ as $\epsilon \rightarrow 0^{+}$and hence in $\mathcal{O}_{C, \omega}\left(\mathbb{R}^{N}\right)$, taking into account that $\mathcal{O}_{C, \omega}\left(\mathbb{R}^{N}\right)$ is the inductive limit of the Fréchet spaces $\left\{\bigcap_{m=1}^{\infty} \mathcal{O}_{n, \omega}^{m}\left(\mathbb{R}^{N}\right)\right\}_{n \in \mathbb{N}}$.

We now prove that $\mathcal{S}_{\omega}\left(\mathbb{R}^{N}\right)$ is a dense subspace of $\mathcal{O}_{M, \omega}\left(\mathbb{R}^{N}\right)$, thereby obtaing by Theorem 3.8 that $\mathcal{O}_{C, \omega}\left(\mathbb{R}^{N}\right)$ is also a dense subspace of $\mathcal{O}_{M, \omega}\left(\mathbb{R}^{N}\right)$. Since $\mathcal{D}_{\omega}\left(\mathbb{R}^{N}\right) \subseteq$ $\mathcal{S}_{\omega}\left(\mathbb{R}^{N}\right)$, it suffices to show only that $\mathcal{D}_{\omega}\left(\mathbb{R}^{N}\right)$ is a dense subspace of $\mathcal{O}_{M, \omega}\left(\mathbb{R}^{N}\right)$. So, fix $f \in \mathcal{O}_{M, \omega}\left(\mathbb{R}^{N}\right)$ and $\phi \in \mathcal{D}_{\omega}\left(\mathbb{R}^{N}\right)$ such that $\phi \equiv 1$ on $\overline{B_{1}(0)}$ and $0 \leq \phi \leq 1$. Then for every $\epsilon>0$ the function $\phi_{\epsilon}(x):=\phi(\epsilon x) f(x)$, for $x \in \mathbb{R}^{N}$, belongs to $\mathcal{D}_{\omega}\left(\mathbb{R}^{N}\right)$ because $\mathcal{O}_{M, \omega}\left(\mathbb{R}^{N}\right) \subseteq \mathcal{E}_{\omega}\left(\mathbb{R}^{N}\right)$. To show that $\varphi_{\epsilon} \rightarrow f$ in $\mathcal{O}_{M, \omega}\left(\mathbb{R}^{N}\right)$, we proceed as follows.

Fix $m \in \mathbb{N}$ and let $M \in \mathbb{N}$ such that $M \geq m L$, where $L \geq 1$ is the constant appearing in formula (2.2). Since $f \in \mathcal{O}_{M, \omega}\left(\mathbb{R}^{N}\right)$, there exist $n(m), n(M) \in \mathbb{N}$ such that $f \in \mathcal{O}_{n(m), \omega}^{m}\left(\mathbb{R}^{N}\right)$ and $f \in \mathcal{O}_{n(M), \omega}^{M}\left(\mathbb{R}^{N}\right)$. Clearly, this implies that $f \in$ $\mathcal{O}_{h, \omega}^{m}\left(\mathbb{R}^{N}\right) \cap \mathcal{O}_{h, \omega}^{M}\left(\mathbb{R}^{N}\right)$ for every $h \geq n:=\max \{n(M), n(m)\}$. Therefore, we can proceed as above to show that for every $\epsilon>0$ we have

$$
\begin{aligned}
r_{m, n+1}\left(\phi_{\epsilon}-f\right) \leq & \sup _{\alpha \in \mathbb{N}_{0}^{N}} \sup _{x \in \mathbb{R}^{N}} \exp \left(-m \varphi_{\omega}^{*}\left(\frac{|\alpha|}{m}\right)\right) \exp (-(n+1) \omega(x)) \\
& \times\left|\partial^{\alpha} f(x)(\phi(\epsilon x)-1)\right|+\epsilon C r_{M, n+1}(f) r_{M, 0}(\phi)
\end{aligned}
$$

Since $f \in \mathcal{O}_{n+1, \omega}^{M}\left(\mathbb{R}^{N}\right)$ and $\phi \in \mathcal{D}_{\omega}\left(\mathbb{R}^{N}\right)$, we have $r_{M, n+1}(f) r_{M, 0}(\phi)<\infty$ and hence, $\epsilon C r_{M, n+1}(f) r_{M, 0}(\phi) \rightarrow 0$ as $\epsilon \rightarrow 0^{+}$. In order to conclude the proof, it then remains to prove that

$$
\sup _{\alpha \in \mathbb{N}_{0}^{N}} \sup _{x \in \mathbb{R}^{N}} \exp \left(-m \varphi_{\omega}^{*}\left(\frac{|\alpha|}{m}\right)\right) \exp (-(n+1) \omega(x))\left|\partial^{\alpha} f(x)(\phi(\epsilon x)-1)\right| \rightarrow 0 \text {, as } \epsilon \rightarrow 0^{+} .
$$


As before, we have $\phi(\epsilon x)-1=0$ whenever $|x| \leq \frac{1}{\epsilon}$. Therefore, for every $\epsilon>0$ we have

$$
\begin{aligned}
& \sup _{\alpha \in \mathbb{N}_{0}^{N}} \sup _{x \in \mathbb{R}^{N}} \exp \left(-m \varphi_{\omega}^{*}\left(\frac{|\alpha|}{m}\right)\right) \exp (-(n+1) \omega(x))\left|\partial^{\alpha} f(x)(\phi(\epsilon x)-1)\right| \\
& \quad \leq r_{m, n}(f) \sup _{|x|>\frac{1}{\epsilon}} \exp (-\omega(x)),
\end{aligned}
$$

where $\sup _{|x|>\frac{1}{\epsilon}} \exp (-\omega(x)) \rightarrow 0$ as $\epsilon \rightarrow 0^{+}$and $r_{m, n}(f)<\infty$. This means that $\phi_{\epsilon} \rightarrow f$ in the Banach space $\mathcal{O}_{n+1, \omega}^{m}\left(\mathbb{R}^{N}\right)$ as $\epsilon \rightarrow 0^{+}$, and hence in the (LB)-space $\bigcup_{h=1}^{\infty} \mathcal{O}_{h, \omega}^{m}\left(\mathbb{R}^{N}\right)$. Since $m \in \mathbb{N}$ is arbitrary and $\mathcal{O}_{M, \omega}\left(\mathbb{R}^{N}\right)$ is the projective limit of the (LB)-spaces $\left\{\bigcup_{h=1}^{\infty} \mathcal{O}_{h, \omega}^{m}\left(\mathbb{R}^{N}\right)\right\}_{m \in \mathbb{N}}$, we can conclude that $\phi_{\epsilon} \rightarrow f$ in the space $\mathcal{O}_{M, \omega}\left(\mathbb{R}^{N}\right)$ as $\epsilon \rightarrow 0^{+}$. This completes the proof.

\section{$4 \mathcal{O}_{M, \omega}\left(\mathbb{R}^{N}\right)$ is the space of multipliers of the spaces $\mathcal{S}_{\omega}\left(\mathbb{R}^{N}\right)$ and $\mathcal{S}_{\omega}^{\prime}\left(\mathbb{R}^{N}\right)$}

The main aim of this section is to prove that $\mathcal{O}_{M, \omega}\left(\mathbb{R}^{N}\right)$ is the space of multipliers of both the spaces $\mathcal{S}_{\omega}\left(\mathbb{R}^{N}\right)$ and $\mathcal{S}_{\omega}^{\prime}\left(\mathbb{R}^{N}\right)$. In order to do this, we first show some preliminary facts.

Lemma 4.1 Let $\omega$ be a non-quasianalytic weight function and $f \in C^{\infty}\left(\mathbb{R}^{N}\right)$. If fg $\in$ $\mathcal{S}_{\omega}\left(\mathbb{R}^{N}\right)$ for every $g \in \mathcal{S}_{\omega}\left(\mathbb{R}^{N}\right)$, then $f \in \mathcal{E}_{\omega}\left(\mathbb{R}^{N}\right)$.

Proof Fix a compact subset $K$ of $\mathbb{R}^{N}$ and $g \in \mathcal{D}_{\omega}\left(\mathbb{R}^{N}\right) \subseteq \mathcal{S}_{\omega}\left(\mathbb{R}^{N}\right)$ such that $g \equiv 1$ on $K$ and $0 \leq g \leq 1$. Then $f g \in \mathcal{S}_{\omega}\left(\mathbb{R}^{N}\right)$ and so, for every $m \in \mathbb{N}$ we have

$$
q_{m, 1}(f g)=\sup _{\alpha \in \mathbb{N}_{0}^{N}} \sup _{x \in \mathbb{R}^{N}}\left|\partial^{\alpha}(f g)(x)\right| \exp \left(-m \varphi_{\omega}^{*}\left(\frac{|\alpha|}{m}\right)\right) \exp (\omega(x))<\infty .
$$

Since $g \equiv 1$ on $K$, it follows that

$$
\begin{aligned}
p_{K, m}(f) & =\sup _{\alpha \in \mathbb{N}_{0}^{N}} \sup _{x \in K}\left|\partial^{\alpha} f(x)\right| \exp \left(-m \varphi_{\omega}^{*}\left(\frac{|\alpha|}{m}\right)\right) \\
& \leq \sup _{\alpha \in \mathbb{N}_{0}^{N}} \sup _{x \in \mathbb{R}^{N}}\left|\partial^{\alpha}(f g)(x)\right| \exp \left(-m \varphi_{\omega}^{*}\left(\frac{|\alpha|}{m}\right)\right) \exp (\omega(x)) \\
& =q_{m, 1}(f g)<\infty .
\end{aligned}
$$

Since $K$ is arbitrary, we can conclude that $f \in \mathcal{E}_{\omega}\left(\mathbb{R}^{N}\right)$. 
Lemma 4.2 Let $\omega$ be a non-quasianalytic weight function. Let $\rho \in \mathcal{D}_{\omega}\left(\mathbb{R}^{N}\right)$ so that supp $\rho \subseteq B_{1}(0)$ and $\left\{x_{j}\right\}_{j \in \mathbb{N}} \subset \mathbb{R}^{N}$ be a sequence such that $\left|x_{j}\right| \geq\left|x_{j-1}\right|+2$ for every $j \geq 2$ and $\left|x_{1}\right|>1$. If we set

$$
g(x):=\sum_{j \in \mathbb{N}} \rho\left(x-x_{j}\right) \exp \left(-j \omega\left(x_{j}\right)\right), \quad x \in \mathbb{R}^{N},
$$

then $g \in \mathcal{S}_{\omega}\left(\mathbb{R}^{N}\right)$.

Proof Since the functions of the sequence $\left\{\rho\left(\cdot-x_{j}\right)\right\}_{j \in \mathbb{N}}$ have disjoint supports, the series on the right of (4.1) converges in $C^{\infty}\left(\mathbb{R}^{N}\right)$ and so, the function $g$ on the left of (4.1) belongs to $C^{\infty}\left(\mathbb{R}^{N}\right)$. On the other hand, the property $(\gamma)$ of $\omega$ implies that $\exp (\omega(x)) \geq \exp (a)(1+|x|)^{b}$ for every $x \in \mathbb{R}^{N}$. Therefore, $g \in \mathcal{S}\left(\mathbb{R}^{N}\right)$, see [13, Proposition 5 , Chap. $4 \S 11$ ]. In order to conclude the proof, we proceed as follows.

Fix $\lambda, \mu>0$ and $x \in \mathbb{R}^{N}$. Then either $x \in B_{1}\left(x_{l}\right)$ for some $j \in \mathbb{N}$ or $x \notin B_{1}\left(x_{l}\right)$ for all $l \in \mathbb{N}$. Accordingly, we have for every $\alpha \in \mathbb{N}_{0}^{N}$ that either

$$
\partial^{\alpha} g(x)=\exp \left(-j \omega\left(x_{j}\right)\right) \partial^{\alpha} \rho\left(x-x_{j}\right)
$$

or $\partial^{\alpha} g(x)=0$. Since $\rho \in \mathcal{D}_{\omega}\left(\mathbb{R}^{N}\right)$, it follows for every $\alpha \in \mathbb{N}_{0}^{N}$ that

$$
\begin{aligned}
& \exp (\mu \omega(x))\left|\partial^{\alpha} g(x)\right| \leq \\
& \quad \leq \exp (\mu \omega(x)) \exp \left(-j \omega\left(x_{j}\right)\right) \exp \left(-\mu K \omega\left(x-x_{j}\right)+\lambda \varphi_{\omega}^{*}\left(\frac{|\alpha|}{\lambda}\right)\right) q_{\lambda, K \mu}(\rho),
\end{aligned}
$$

where $K$ is the constant appearing in the property $(\alpha)$ of $\omega$. But, we have

$$
\omega(x)=\omega\left(\left(x-x_{j}\right)+x_{j}\right) \leq K\left(\omega\left(x-x_{j}\right)+\omega\left(x_{j}\right)+1\right) .
$$

So, by (4.2) we obtain for every $\alpha \in \mathbb{N}_{0}^{N}$ that

$$
\begin{aligned}
\exp (\mu \omega(x))\left|\partial^{\alpha} g(x)\right| \leq & e^{K} \exp \left(K \mu \omega\left(x-x_{j}\right)+K \mu \omega\left(x_{j}\right)\right) \\
& \quad \times \exp \left(-j \omega\left(x_{j}\right)\right) \exp \left(-\mu K \omega\left(x-x_{j}\right)+\lambda \varphi_{\omega}^{*}\left(\frac{|\alpha|}{\lambda}\right)\right) q_{\lambda, K \mu}(\rho) \\
= & e^{K} \exp \left((K \mu-j) \omega\left(x_{j}\right)\right) \exp \left(\lambda \varphi_{\omega}^{*}\left(\frac{|\alpha|}{\lambda}\right)\right) q_{\lambda, K \mu}(\rho)
\end{aligned}
$$

and hence,

$$
\exp \left(-\lambda \varphi_{\omega}^{*}\left(\frac{|\alpha|}{\lambda}\right)\right) \exp (\mu \omega(x))\left|\partial^{\alpha} g(x)\right| \leq e^{K} \exp \left((K \mu-j) \omega\left(x_{j}\right)\right) q_{\lambda, K \mu}(\rho)
$$

Since $\exp \left((K \mu-j) \omega\left(x_{j}\right)\right) \leq 1$ whenever $j \geq K \mu$ and $x$ is arbitrary, it follows that

$$
q_{\lambda, \mu}(g) \leq e^{K} \max _{j<K \mu} \exp \left((K \mu-j) \omega\left(x_{j}\right)\right) q_{\lambda, K \mu}(\rho)<\infty .
$$


But, $\lambda$ and $\mu$ are also arbitrary. So, we can conclude that $g \in \mathcal{S}_{\omega}\left(\mathbb{R}^{N}\right)$.

Remark 4.3 Let $\omega$ be a non-quasianalytic weight function. Let $\rho \in \mathcal{D}_{\omega}\left(\mathbb{R}^{N}\right)$ so that supp $\rho \subseteq B_{1}(0)$ and $\left\{x_{j}\right\}_{j \in \mathbb{N}} \subset \mathbb{R}^{N}$ be a sequence such that $\left|x_{j}\right| \geq\left|x_{j-1}\right|+2$ for every $j \geq 2$ and $\left|x_{1}\right|>1$. If $h \in \mathcal{S}_{\omega}\left(\mathbb{R}^{N}\right)$ and $g(x):=\sum_{j \in \mathbb{N}} h\left(x_{j}\right) \rho\left(x-x_{j}\right)$ for each $x \in \mathbb{R}^{N}$, then $g \in \mathcal{S}_{\omega}\left(\mathbb{R}^{N}\right)$. The proof follows by arguing as in the proof of Lemma 4.2, after having observed that $\sup _{x \in \mathbb{R}^{N}}|h(x)| \exp (j \omega(x))<\infty$ for each $j \in \mathbb{N}$.

We can now state and prove that $\mathcal{O}_{M, \omega}\left(\mathbb{R}^{N}\right)$ is the space of multipliers of $\mathcal{S}_{\omega}\left(\mathbb{R}^{N}\right)$.

Theorem 4.4 Let $\omega$ be a non-quasianalytic weight function and $f \in C^{\infty}\left(\mathbb{R}^{N}\right)$. Then the following properties are equivalent.

(1) $f \in \mathcal{O}_{M, \omega}\left(\mathbb{R}^{N}\right)$.

(2) For every $g \in \mathcal{S}_{\omega}\left(\mathbb{R}^{N}\right)$ we have $f g \in \mathcal{S}_{\omega}\left(\mathbb{R}^{N}\right)$.

Moreover, if $f \in \mathcal{O}_{M, \omega}\left(\mathbb{R}^{N}\right)$, then the linear operator $M_{f}: \mathcal{S}_{\omega}\left(\mathbb{R}^{N}\right) \rightarrow \mathcal{S}_{\omega}\left(\mathbb{R}^{N}\right)$ defined by $M_{f}(g):=f g$, for $g \in \mathcal{S}_{\omega}\left(\mathbb{R}^{N}\right)$, is continuous.

Proof $(1) \Rightarrow(2)$. Fix $g \in \mathcal{S}_{\omega}\left(\mathbb{R}^{N}\right)$. Then $f g \in C^{\infty}\left(\mathbb{R}^{N}\right)$. Moreover, for every $\alpha \in \mathbb{N}_{0}^{N}$ and $x \in \mathbb{R}^{N}$ we have

$$
\left|\partial^{\alpha}(f g)(x)\right| \leq \sum_{\gamma \leq \alpha}\left(\begin{array}{l}
\alpha \\
\gamma
\end{array}\right)\left|\partial^{\gamma} f(x)\right|\left|\partial^{\alpha-\gamma} g(x)\right| .
$$

Fixed $m \in \mathbb{N}$, let $M \in \mathbb{N}$ so that $M \geq L m$, where $L \geq 1$ is the constant appearing in formula (2.2). Since $f \in \mathcal{O}_{M, \omega}\left(\mathbb{R}^{N}\right)$, there exist $n \in \bar{N}$ and $C>0$ such that for every $\gamma \in \mathbb{N}_{0}^{N}$ and $x \in \mathbb{R}^{N}$ we have

$$
\left|\partial^{\gamma} f(x)\right| \leq C \exp \left(n \omega(x)+M \varphi_{\omega}^{*}\left(\frac{|\gamma|}{M}\right)\right) .
$$

On the other hand, $g \in \mathcal{S}_{\omega}\left(\mathbb{R}^{N}\right)$. So, setting $M^{\prime}:=\max \{M, n+m\}$ and recalling that $\varphi_{\omega}^{*}(t) / t$ is an increasing function in $(0, \infty)$, we have for every $\delta \in \mathbb{N}_{0}^{N}$ and $x \in \mathbb{R}^{N}$ that

$$
\begin{aligned}
\left|\partial^{\delta} g(x)\right| & \leq q_{M^{\prime}, M^{\prime}}(g) \exp \left(M^{\prime} \varphi_{\omega}^{*}\left(\frac{|\delta|}{M^{\prime}}\right)-M^{\prime} \omega(x)\right) \\
& \leq q_{M^{\prime}, M^{\prime}}(g) \exp \left(M \varphi_{\omega}^{*}\left(\frac{|\delta|}{M}\right)-M^{\prime} \omega(x)\right) .
\end{aligned}
$$

By combining (4.3), (4.4) and (4.5), we obtain for every $\alpha \in \mathbb{N}_{0}^{N}$ and $x \in \mathbb{R}^{N}$ that

$$
\begin{aligned}
& \exp (m \omega(x))\left|\partial^{\alpha}(f g)(x)\right| \leq \\
& \quad \leq \sum_{\gamma \leq \alpha}\left(\begin{array}{l}
\alpha \\
\gamma
\end{array}\right) C \exp ((n+m) \omega(x)) \exp \left(M \varphi_{\omega}^{*}\left(\frac{|\gamma|}{M}\right)\right)\left|\partial^{\alpha-\gamma} g(x)\right|
\end{aligned}
$$




$$
\begin{aligned}
\leq & C \sum_{\gamma \leq \alpha}\left(\begin{array}{l}
\alpha \\
\gamma
\end{array}\right) \exp ((n+m) \omega(x)) \exp \left(M \varphi_{\omega}^{*}\left(\frac{|\gamma|}{M}\right)\right) q_{M^{\prime}, M^{\prime}}(g) \\
& \times \exp \left(M \varphi_{\omega}^{*}\left(\frac{|\alpha-\gamma|}{M}\right)-M^{\prime} \omega(x)\right) \\
\leq & C q_{M^{\prime}, M^{\prime}}(g) \exp \left(\left(n+m-M^{\prime}\right) \omega(x)\right) \sum_{\gamma \leq \alpha}\left(\begin{array}{l}
\alpha \\
\gamma
\end{array}\right) \exp \left(M \varphi_{\omega}^{*}\left(\frac{|\gamma|}{M}\right)\right) \times \\
& \times \exp \left(M \varphi_{\omega}^{*}\left(\frac{|\alpha-\gamma|}{M}\right)\right) .
\end{aligned}
$$

Using inequality (2.7) and taking in mind that $\sup _{x \in \mathbb{R}^{N}} \exp \left(\left(n+m-M^{\prime}\right) \omega(x)\right)<\infty$, it follows for every $\alpha \in \mathbb{N}_{0}^{N}$ and $x \in \mathbb{R}^{N}$ that

$$
\exp (m \omega(x))\left|\partial^{\alpha}(f g)(x)\right| \leq C q_{M^{\prime}, M^{\prime}}(g) 2^{|\alpha|} \exp \left(M \varphi_{\omega}^{*}\left(\frac{|\alpha|}{M}\right)\right)
$$

Since $M \geq m L$, applying formula (2.6) we obtain via (4.6) that for every $\alpha \in \mathbb{N}_{0}^{N}$ and $x \in \mathbb{R}^{N}$

$$
\exp (m \omega(x))\left|\partial^{\alpha}(f g)(x)\right| \leq C q_{M^{\prime}, M^{\prime}}(g) \exp \left(m \varphi_{\omega}^{*}\left(\frac{|\alpha|}{m}\right)\right)
$$

and so

$$
q_{m, m}(f g) \leq C \exp (m L) q_{M^{\prime}, M^{\prime}}(g)<\infty .
$$

The thesis follows from the arbitrarity of $m \in \mathbb{N}$.

$(2) \Rightarrow(1)$. We first observe that by Lemma 4.1 we have $f \in \mathcal{E}_{\omega}\left(\mathbb{R}^{N}\right)$. We now suppose that $f \notin \mathcal{O}_{M, \omega}\left(\mathbb{R}^{N}\right)$. Then by Proposition 3.3(1) there exists $m \in \mathbb{N}$ such that for each $C, R>0$ and $n \in \mathbb{N}$ there exist $x \in \mathbb{R}^{N}$ with $|x|>R$ and $\alpha \in \mathbb{N}_{0}^{N}$ such that

$$
\left|\partial^{\alpha} f(x)\right| \geq C \exp \left(n \omega(x)+m \varphi_{\omega}^{*}\left(\frac{|\alpha|}{m}\right)\right) .
$$

So, we can choose two sequences $\left\{\alpha_{j}\right\}_{j \in \mathbb{N}} \subset \mathbb{N}_{0}^{N}$ and $\left\{x_{j}\right\}_{j \in \mathbb{N}} \subset \mathbb{R}^{N}$ with $\left|x_{j+1}\right|>$ $\left|x_{j}\right|+2$ for all $j \in \mathbb{N}$ and $\left|x_{1}\right|>1$ such that

$$
\left|\partial^{\alpha_{j}} f\left(x_{j}\right)\right| \geq \exp \left(j \omega\left(x_{j}\right)+m \varphi_{\omega}^{*}\left(\frac{\left|\alpha_{j}\right|}{m}\right)\right) .
$$

Let $\rho \in \mathcal{D}_{\omega}\left(\mathbb{R}^{N}\right) \subset \mathcal{S}_{\omega}\left(\mathbb{R}^{N}\right)$ such that supp $\rho \subseteq B_{1}(0)$ and $\rho \equiv 1$ on $B_{r}(0)$ for some $0<r<1$. For every $x \in \mathbb{R}^{N}$ let

$$
g(x):=\sum_{j \in \mathbb{N}} \rho\left(x-x_{j}\right) \exp \left(-j \omega\left(x_{j}\right)\right)
$$


Then by Lemma 4.2 we have $g \in \mathcal{S}_{\omega}\left(\mathbb{R}^{N}\right)$ and so, $q_{m, m}(f g)<\infty$. In particular, we have that

$$
\sup _{j \in \mathbb{N}}\left|\partial^{\alpha_{j}}(f g)\left(x_{j}\right)\right| \exp \left(m \omega\left(x_{j}\right)-m \varphi_{\omega}^{*}\left(\frac{\left|\alpha_{j}\right|}{m}\right)\right) \leq q_{m, m}(f g)<\infty
$$

On the other hand, for every $j \in \mathbb{N}$ we have $g \equiv \exp \left(-j \omega\left(x_{j}\right)\right)$ in $\bar{B}_{r}\left(x_{j}\right)$. Accordingly, for every $j \in \mathbb{N}, x \in \bar{B}_{r}\left(x_{j}\right)$ and $\alpha \in \mathbb{N}_{0}^{N}$ we have

$$
\partial^{\alpha}(f g)(x)=\exp \left(-j \omega\left(x_{j}\right)\right) \partial^{\alpha} f(x) .
$$

Therefore, by (4.8) and (4.9) it follows that

$$
\begin{aligned}
q_{m, m}(f g) & \geq \sup _{j \in \mathbb{N}}\left|\partial^{\alpha_{j}} f\left(x_{j}\right)\right| \exp \left(m \omega\left(x_{j}\right)-m \varphi_{\omega}^{*}\left(\frac{\left|\alpha_{j}\right|}{m}\right)\right) \exp \left(-j \omega\left(x_{j}\right)\right) \\
& \geq \sup _{j \in \mathbb{N}} \exp \left(m \omega\left(x_{j}\right)\right),
\end{aligned}
$$

thereby implying that $\sup _{j \in \mathbb{N}} \exp \left(m \omega\left(x_{j}\right)\right)<\infty$. But, $\lim _{j \rightarrow \infty} \exp \left(m \omega\left(x_{j}\right)\right)=+\infty$ and hence, $\sup _{j \in \mathbb{N}} \exp \left(m \omega\left(x_{j}\right)\right)=\infty$. This is a contradiction.

Fix any $f \in \mathcal{O}_{M, \omega}\left(\mathbb{R}^{N}\right)$. Then the operator $M_{f}: \mathcal{S}_{\omega}\left(\mathbb{R}^{N}\right) \rightarrow \mathcal{S}_{\omega}\left(\mathbb{R}^{N}\right)$ is well defined by the proof above. Actually, $M_{f}$ is also continuous as follows directly from (4.7).

Remark 4.5 (a) Let $f \in \mathcal{S}_{\omega}\left(\mathbb{R}^{N}\right)$. Then $f g \in \mathcal{S}_{\omega}\left(\mathbb{R}^{N}\right)$ for every $g \in \mathcal{S}_{\omega}\left(\mathbb{R}^{N}\right)$. So, by Theorem 4.4 it follows that $f \in \mathcal{O}_{M, \omega}\left(\mathbb{R}^{N}\right)$.

(b) Let $f \in \mathcal{E}_{\omega}\left(\mathbb{R}^{N}\right)$. Then $f g \in \mathcal{S}_{\omega}\left(\mathbb{R}^{N}\right)$ for every $g \in \mathcal{S}_{\omega}\left(\mathbb{R}^{N}\right)$ if and only if $\left(\partial^{\alpha} f\right) g \in \mathcal{S}_{\omega}\left(\mathbb{R}^{N}\right)$ for every $g \in \mathcal{S}_{\omega}\left(\mathbb{R}^{N}\right)$ and $\alpha \in \mathbb{N}_{0}^{N}$.

Indeed, the condition on the right clearly implies the condition on the left. Conversely, the assumption $f g \in \mathcal{S}_{\omega}\left(\mathbb{R}^{N}\right)$ for every $g \in \mathcal{S}_{\omega}\left(\mathbb{R}^{N}\right)$ implies for every $j=1, \ldots, N$ that

$$
\left(\partial_{j} f\right) g=\partial_{j}(f g)-f\left(\partial_{j} g\right) \in \mathcal{S}_{\omega}\left(\mathbb{R}^{N}\right) .
$$

So, proceeding by induction the result follows.

Consequently, by Theorem 4.4 we can conclude that for fixed $f \in \mathcal{O}_{M, \omega}\left(\mathbb{R}^{N}\right)$ and $\alpha \in \mathbb{N}_{0}^{N}$ the function $\partial^{\alpha} f \in \mathcal{O}_{M, \omega}\left(\mathbb{R}^{N}\right)$ too, and the linear operator $M_{\partial^{\alpha} f}$ : $\mathcal{S}_{\omega}\left(\mathbb{R}^{N}\right) \rightarrow \mathcal{S}_{\omega}\left(\mathbb{R}^{N}\right)$ is continuous.

Finally, we show that $\mathcal{O}_{M, \omega}\left(\mathbb{R}^{N}\right)$ is also the space of multipliers of $\mathcal{S}_{\omega}^{\prime}\left(\mathbb{R}^{N}\right)$.

Theorem 4.6 Let $\omega$ be a non-quasianalytic weight function and $f \in \mathcal{E}_{\omega}\left(\mathbb{R}^{N}\right)$. Then the following properties are equivalent.

(1) $f \in \mathcal{O}_{M, \omega}\left(\mathbb{R}^{N}\right)$.

(2) For every $T \in \mathcal{S}_{\omega}^{\prime}\left(\mathbb{R}^{N}\right)$ we have $f T \in \mathcal{S}_{\omega}^{\prime}\left(\mathbb{R}^{N}\right)$. 
Moreover, if $f \in \mathcal{O}_{M, \omega}\left(\mathbb{R}^{N}\right)$, then the linear operator $\mathcal{M}_{f}: \mathcal{S}_{\omega}^{\prime}\left(\mathbb{R}^{N}\right) \rightarrow \mathcal{S}_{\omega}^{\prime}\left(\mathbb{R}^{N}\right)$ defined by $\mathcal{M}_{f}(T):=f T$, for $T \in \mathcal{S}_{\omega}^{\prime}\left(\mathbb{R}^{N}\right)$, is continuous.

Proof $(1) \Rightarrow(2)$. Fix $T \in \mathcal{S}_{\omega}^{\prime}\left(\mathbb{R}^{N}\right)$. By Theorem 4.4 the linear operator $M_{f}: \mathcal{S}_{\omega}\left(\mathbb{R}^{N}\right) \rightarrow$ $\mathcal{S}_{\omega}\left(\mathbb{R}^{N}\right)$ given by $M_{f}(g)=f g$, for $g \in \mathcal{S}_{\omega}\left(\mathbb{R}^{N}\right)$, is continuous and so, the linear functional $T \circ M_{f}: \mathcal{S}_{\omega}\left(\mathbb{R}^{N}\right) \rightarrow \mathbb{C}$ is also continuous. Accordingly, $T \circ M_{f} \in \mathcal{S}_{\omega}^{\prime}\left(\mathbb{R}^{N}\right)$. But, for every $g \in \mathcal{S}_{\omega}\left(\mathbb{R}^{N}\right)$ we have

$$
\left(T \circ M_{f}\right)(g)=\left\langle T, M_{f}(g)\right\rangle=\langle T, f g\rangle=\langle f T, g\rangle .
$$

Therefore, $f T=T \circ M_{f} \in \mathcal{S}_{\omega}^{\prime}\left(\mathbb{R}^{N}\right)$. This completes the proof.

(2) $\Rightarrow(1)$. Suppose for every $T \in \mathcal{S}_{\omega}^{\prime}\left(\mathbb{R}^{N}\right)$ that $f T \in \mathcal{S}_{\omega}^{\prime}\left(\mathbb{R}^{N}\right)$. Then for a fixed $g \in \mathcal{S}_{\omega}\left(\mathbb{R}^{N}\right)$ the linear functional $L: \mathcal{S}_{\omega}^{\prime}\left(\mathbb{R}^{N}\right) \rightarrow \mathbb{C}$ defined by $L(T):=\langle f T, g\rangle$, for $T \in \mathcal{S}_{\omega}^{\prime}\left(\mathbb{R}^{N}\right)$, is continuous and so, $L \in \mathcal{S}_{\omega}^{\prime \prime}\left(\mathbb{R}^{N}\right)$. Since $\mathcal{S}_{\omega}\left(\mathbb{R}^{N}\right)$ is reflexive, there exists $h \in \mathcal{S}_{\omega}\left(\mathbb{R}^{N}\right)$ such that $L(T)=\langle T, h\rangle$ for every $T \in \mathcal{S}_{\omega}^{\prime}\left(\mathbb{R}^{N}\right)$, i.e., $\langle f T, g\rangle=$ $\langle T, h\rangle$ for every $T \in \mathcal{S}_{\omega}^{\prime}\left(\mathbb{R}^{N}\right)$. Accordingly, for every $T \in \mathcal{D}_{\omega}\left(\mathbb{R}^{N}\right) \subseteq \mathcal{S}_{\omega}^{\prime}\left(\mathbb{R}^{N}\right)$ we have $\langle f T, g\rangle=\langle T, h\rangle$. Since for every $T \in \mathcal{D}_{\omega}\left(\mathbb{R}^{N}\right)$ we have $\langle f T, g\rangle=\langle T, f g\rangle$ and $\mathcal{D}_{\omega}\left(\mathbb{R}^{N}\right)$ is a dense subspace of $\mathcal{S}_{\omega}\left(\mathbb{R}^{N}\right)$, it follows that $f g=h \in \mathcal{S}_{\omega}\left(\mathbb{R}^{N}\right)$. So, as $g \in \mathcal{S}_{\omega}\left(\mathbb{R}^{N}\right)$ is arbitrary, by Theorem 4.4 we can conclude that $f \in \mathcal{O}_{M, \omega}\left(\mathbb{R}^{N}\right)$.

Fix any $f \in \mathcal{O}_{M, \omega}\left(\mathbb{R}^{N}\right)$. Then for every $g \in \mathcal{S}_{\omega}\left(\mathbb{R}^{N}\right)$ and $T \in \mathcal{S}_{\omega}^{\prime}\left(\mathbb{R}^{N}\right)$ we have

$$
\langle f T, g\rangle=\langle T, f g\rangle \text {. }
$$

This means that the linear operator $\mathcal{M}_{f}: \mathcal{S}_{\omega}^{\prime}\left(\mathbb{R}^{N}\right) \rightarrow \mathcal{S}_{\omega}^{\prime}\left(\mathbb{R}^{N}\right)$ is the transpose of the continuous linear operator $M_{f}: \mathcal{S}_{\omega}\left(\mathbb{R}^{N}\right) \rightarrow \mathcal{S}_{\omega}\left(\mathbb{R}^{N}\right)$. Therefore, the linear operator $\mathcal{M}_{f}: \mathcal{S}_{\omega}^{\prime}\left(\mathbb{R}^{N}\right) \rightarrow \mathcal{S}_{\omega}^{\prime}\left(\mathbb{R}^{N}\right)$ is necessarily continuous.

\section{Other topologies on $\mathcal{O}_{M, \omega}\left(\mathbb{R}^{N}\right)$}

In this section we show that the space $\mathcal{O}_{M, \omega}\left(\mathbb{R}^{N}\right)$ can be naturally endowed with other lc-topologies. We also compare these lc-topologies with each other and with the projective lc-topology defined on $\mathcal{O}_{M, \omega}\left(\mathbb{R}^{N}\right)$ by the spectrum $\left\{\cup_{n=1}^{\infty} \mathcal{O}_{n, \omega}^{m}\left(\mathbb{R}^{N}\right)\right\}_{m \in \mathbb{N}}$.

We begin by giving another useful chartacterization of the space $\mathcal{O}_{M, \omega}\left(\mathbb{R}^{N}\right)$.

Theorem 5.1 Let $\omega$ be a non-quasianalytic weight function and $f \in C^{\infty}\left(\mathbb{R}^{N}\right)$. Then the following properties are equivalent.

(1) $f \in \mathcal{O}_{M, \omega}\left(\mathbb{R}^{N}\right)$.

(2) For every $g \in \mathcal{S}_{\omega}\left(\mathbb{R}^{N}\right)$ and $m \in \mathbb{N}$ we have

$$
q_{m, g}(f):=\sup _{\alpha \in \mathbb{N}_{0}^{N}} \sup _{x \in \mathbb{R}^{N}}\left|g(x) \| \partial^{\alpha} f(x)\right| \exp \left(-m \varphi_{\omega}^{*}\left(\frac{|\alpha|}{m}\right)\right)<\infty .
$$

Proof $(1) \Rightarrow(2)$. Since $f \in \mathcal{O}_{M, \omega}\left(\mathbb{R}^{N}\right)$, we have for every $m \in \mathbb{N}$ that there exist $C>0$ and $n \in \mathbb{N}$ such that for every $\alpha \in \mathbb{N}_{0}^{N}$ and $x \in \mathbb{R}^{N}$ the following inequality is 
satisfied

$$
\left|\partial^{\alpha} f(x)\right| \leq C \exp \left(n \omega(x)+m \varphi_{\omega}^{*}\left(\frac{|\alpha|}{m}\right)\right) .
$$

Therefore, for any fixed $g \in \mathcal{S}_{\omega}\left(\mathbb{R}^{N}\right)$, it follows for every $m \in \mathbb{N}, \alpha \in \mathbb{N}_{0}^{N}$ and $x \in \mathbb{R}^{N}$ that

$$
|g(x)|\left|\partial^{\alpha} f(x)\right| \exp \left(-m \varphi_{\omega}^{*}\left(\frac{|\alpha|}{m}\right)\right) \leq C \exp (n \omega(x))|g(x)|,
$$

with $n \in \mathbb{N}$ depending only of $m$. This implies that

$$
q_{m, g}(f) \leq C \sup _{x \in \mathbb{R}^{N}} \exp (n \omega(x))|g(x)|<\infty .
$$

Since $g \in \mathcal{S}_{\omega}\left(\mathbb{R}^{N}\right)$ is arbitrary, the thesis follows.

$(2) \Rightarrow(1)$. We first show that $f \in \mathcal{E}_{\omega}\left(\mathbb{R}^{N}\right)$. So, fixed any compact subset $K$ of $\mathbb{R}^{N}$, let $g \in \mathcal{D}_{\omega}\left(\mathbb{R}^{N}\right)$ such that $g \equiv 1$ on $K$. Then it follows for every $m \in \mathbb{N}$ that

$$
\begin{aligned}
p_{K, m}(f) & =\sup _{x \in K} \sup _{\alpha \in \mathbb{N}_{0}^{N}}\left|\partial^{\alpha} f(x)\right| \exp \left(-m \varphi_{\omega}^{*}\left(\frac{|\alpha|}{m}\right)\right) \\
& \leq \sup _{x \in K} \sup _{\alpha \in \mathbb{N}_{0}^{N}}|g(x)|\left|\partial^{\alpha} f(x)\right| \exp \left(-m \varphi_{\omega}^{*}\left(\frac{|\alpha|}{m}\right)\right) \\
& \leq q_{m, g}(f)<\infty .
\end{aligned}
$$

Since $K$ is an arbitrary compact subset of $\mathbb{R}^{N}$, we can conclude that $f \in \mathcal{E}_{\omega}\left(\mathbb{R}^{N}\right)$.

Suppose that $f \notin \mathcal{O}_{M, \omega}\left(\mathbb{R}^{N}\right)$. Since $f \in \mathcal{E}_{\omega}\left(\mathbb{R}^{N}\right)$, it follows from Proposition 3.3(1) that there exists $m \in \mathbb{N}$ such that for each $C, R>0$ and $n \in \mathbb{N}$ there exist $x \in \mathbb{R}^{N}$ with $|x|>R$ and $\alpha \in \mathbb{N}_{0}^{N}$ such that

$$
\left|\partial^{\alpha} f(x)\right| \geq C \exp \left(n \omega(x)+m \varphi_{\omega}^{*}\left(\frac{|\alpha|}{m}\right)\right) .
$$

So, we can choose two sequences $\left\{\alpha_{j}\right\}_{j \in \mathbb{N}} \subset \mathbb{N}_{0}^{N}$ and $\left\{x_{j}\right\}_{j \in \mathbb{N}} \subset \mathbb{R}^{N}$ with $\left|x_{j+1}\right|>$ $\left|x_{j}\right|+2$ for all $j \in \mathbb{N}$ and $\left|x_{1}\right|>1$ such that

$$
\left|\partial^{\alpha_{j}} f\left(x_{j}\right)\right| \geq \exp \left(j \omega\left(x_{j}\right)+m \varphi_{\omega}^{*}\left(\frac{\left|\alpha_{j}\right|}{m}\right)\right) .
$$

Next, let $\rho \in \mathcal{D}_{\omega}\left(\mathbb{R}^{N}\right) \subset \mathcal{S}_{\omega}\left(\mathbb{R}^{N}\right)$ such that supp $\rho \subseteq B_{1}(0)$ and $\rho \equiv 1$ on $B_{r}(0)$ for some $0<r<1$. For every $x \in \mathbb{R}^{N}$ let

$$
g(x):=\sum_{j \in \mathbb{N}} \rho\left(x-x_{j}\right) \exp \left(-j \omega\left(x_{j}\right)\right)
$$


Then by Lemma 4.2 we have $g \in \mathcal{S}_{\omega}\left(\mathbb{R}^{N}\right)$ and hence, it necessarily holds that $q_{m, g}(f)<\infty$. In particular, we have that

$$
\sup _{j \in \mathbb{N}}\left|g\left(x_{j}\right)\right|\left|\partial^{\alpha_{j}} f\left(x_{j}\right)\right| \exp \left(m \omega\left(x_{j}\right)-m \varphi_{\omega}^{*}\left(\frac{\left|\alpha_{j}\right|}{m}\right)\right) \leq q_{m, g}(f)<\infty .
$$

But, $g\left(x_{j}\right)=\exp \left(-j \omega\left(x_{j}\right)\right)$ for every $j \in \mathbb{N}$. So, it follows by (5.3) and (5.4) that

$$
\begin{aligned}
q_{m, g}(f) & \geq \sup _{j \in \mathbb{N}} \exp \left(-j \omega\left(x_{j}\right)\left|\partial^{\alpha_{j}} f\left(x_{j}\right)\right| \exp \left(m \omega\left(x_{j}\right)-m \varphi_{\omega}^{*}\left(\frac{\left|\alpha_{j}\right|}{m}\right)\right)\right. \\
& \geq \sup _{j \in \mathbb{N}} \exp \left(m \omega\left(x_{j}\right)\right),
\end{aligned}
$$

thereby implying that $\sup _{j \in \mathbb{N}} \exp \left(m \omega\left(x_{j}\right)\right)<\infty$. This is a contradiction because $\lim _{j \rightarrow \infty} \exp \left(m \omega\left(x_{j}\right)\right)=\infty$. Hence, $f$ necessarily belongs to $\mathcal{O}_{M, \omega}\left(\mathbb{R}^{N}\right)$.

Theorem 5.1 implies that the set $\left\{q_{m, g}\right\}_{m \in \mathbb{N}, g \in \mathcal{S}_{\omega}\left(\mathbb{R}^{N}\right)}$ forms a fundamental system of norms on $\mathcal{O}_{M, \omega}\left(\mathbb{R}^{N}\right)$. Denote by $\tau$ the Hausdorff lc-topology on $\mathcal{O}_{M, \omega}\left(\mathbb{R}^{N}\right)$ generated by $\left\{q_{m, g}\right\}_{m \in \mathbb{N}, g \in \mathcal{S}_{\omega}\left(\mathbb{R}^{N}\right)}$.

We now collect some properties of the Hausdorff lc-space $\left(\mathcal{O}_{M, \omega}\left(\mathbb{R}^{N}\right), \tau\right)$.

Theorem 5.2 Let $\omega$ be a non-quasianalytic weight function. Then the following properties are satisfied.

(1) The inclusion

$$
\left(\mathcal{O}_{M, \omega}\left(\mathbb{R}^{N}\right), \tau\right) \hookrightarrow \mathcal{E}_{\omega}\left(\mathbb{R}^{N}\right)
$$

is continuous with dense range.

(2) $\left(\mathcal{O}_{M, \omega}\left(\mathbb{R}^{N}\right), \tau\right)$ is a complete lc-space.

Proof (1) The continuity of the inclusion $\left(\mathcal{O}_{M, \omega}\left(\mathbb{R}^{N}\right), \tau\right) \hookrightarrow \mathcal{E}_{\omega}\left(\mathbb{R}^{N}\right)$ follows by repeating the arguments at the beginning of the proof of Theorem $5.1(2) \Rightarrow(1)$, i.e., of (5.2). On the other hand, the facts that the inclusion $\mathcal{D}_{\omega}\left(\mathbb{R}^{N}\right) \hookrightarrow \mathcal{E}_{\omega}\left(\mathbb{R}^{N}\right)$ has dense range (see Remark 2.6(3)) and $\mathcal{D}_{\omega}\left(\mathbb{R}^{N}\right) \subseteq \mathcal{O}_{M, \omega}\left(\mathbb{R}^{N}\right)$ (see Remark 3.2(1)) clearly imply that the inclusion $\left(\mathcal{O}_{M, \omega}\left(\mathbb{R}^{N}\right), \tau\right) \hookrightarrow \mathcal{E}_{\omega}\left(\mathbb{R}^{N}\right)$ has dense range too.

(2) Let $\left\{f_{i}\right\}_{i \in I}$ be a Cauchy net in $\left(\mathcal{O}_{M, \omega}\left(\mathbb{R}^{N}\right), \tau\right)$. Since the inclusion $\left(\mathcal{O}_{M, \omega}\right.$ $\left.\left(\mathbb{R}^{N}\right), \tau\right) \hookrightarrow C^{\infty}\left(\mathbb{R}^{N}\right)$ is continuous as it is easy to prove, it follows that $\left\{f_{i}\right\}_{i \in I}$ is also a Cauchy net in $C^{\infty}\left(\mathbb{R}^{N}\right)$. But, $C^{\infty}\left(\mathbb{R}^{N}\right)$ is a Fréchet space and hence, a complete lc-space. So, there exists $f \in C^{\infty}\left(\mathbb{R}^{N}\right)$ such that $f_{i} \rightarrow f$ in $C^{\infty}\left(\mathbb{R}^{N}\right)$. We claim that $f_{i} \rightarrow f$ in $\left(\mathcal{O}_{M, \omega}\left(\mathbb{R}^{N}\right), \tau\right)$. To see this, we fix $g \in \mathcal{S}_{\omega}\left(\mathbb{R}^{N}\right), m \in \mathbb{N}$ and $\epsilon>0$. Since $\left\{f_{i}\right\}_{i \in I}$ is a Cauchy net in $\left(\mathcal{O}_{M, \omega}\left(\mathbb{R}^{N}\right), \tau\right)$, there exists $i_{0} \in I$ such that for every $i, i^{\prime} \geq i_{0}$ we have

$$
q_{m, g}\left(f_{i}-f_{i^{\prime}}\right)=\sup _{x \in \mathbb{R}^{N}} \sup _{\alpha \in \mathbb{N}_{0}^{n}}\left|g(x) \| \partial^{\alpha}\left(f_{i}-f_{i^{\prime}}\right)(x)\right| \exp \left(-m \varphi_{\omega}^{*}\left(\frac{|\alpha|}{m}\right)\right)<\epsilon,
$$


i.e., for every $i, i^{\prime} \geq i_{0}, \alpha \in \mathbb{N}_{0}^{N}$ and $x \in \mathbb{R}^{N}$ we have

$$
|g(x)|\left|\partial^{\alpha}\left(f_{i}-f_{i^{\prime}}\right)(x)\right|<\epsilon \exp \left(m \varphi_{\omega}^{*}\left(\frac{|\alpha|}{m}\right)\right) .
$$

Since $f_{i} \rightarrow f$ in $C^{\infty}\left(\mathbb{R}^{N}\right)$ implies that $\partial^{\alpha} f_{i} \rightarrow \partial^{\alpha} f$ pointwise in $\mathbb{R}^{N}$ for every $\alpha \in \mathbb{N}_{0}^{N}$, by letting $i^{\prime}$ to infty it follows for every $i \geq i_{0}, \alpha \in \mathbb{N}_{0}^{N}$ and $x \in \mathbb{R}^{N}$ that

$$
|g(x)|\left|\partial^{\alpha}\left(f_{i}-f\right)(x)\right|<\epsilon \exp \left(m \varphi_{\omega}^{*}\left(\frac{|\alpha|}{m}\right)\right)
$$

and so,

$$
\begin{aligned}
|g(x)|\left|\partial^{\alpha} f(x)\right| & \leq|g(x)|\left|\partial^{\alpha}\left(f-f_{i_{0}}\right)(x)\right|+|g(x)|\left|\partial^{\alpha} f_{i_{0}}(x)\right| \\
& <\epsilon \exp \left(m \varphi_{\omega}^{*}\left(\frac{|\alpha|}{m}\right)\right)+|g(x)|\left|\partial^{\alpha} f_{i_{0}}(x)\right|
\end{aligned}
$$

Accordingly, we have for every $i \geq i_{0}$ that

$$
q_{m, g}\left(f_{i}-f\right) \leq \epsilon \text { and } q_{m, g}(f) \leq \epsilon+q_{m, g}\left(f_{i_{0}}\right) .
$$

Since $g \in \mathcal{S}_{\omega}\left(\mathbb{R}^{N}\right)$ and $m \in \mathbb{N}$ are arbitrary, this shows via Theorem 5.1 that $f \in$ $\mathcal{O}_{M, \omega}\left(\mathbb{R}^{N}\right)$ and that $f_{i} \rightarrow f$ in $\left(\mathcal{O}_{M, \omega}\left(\mathbb{R}^{N}\right), \tau\right)$.

We recall that the sequence $\left\{\cup_{n=1}^{\infty} \mathcal{O}_{n, \omega}^{m}\left(\mathbb{R}^{N}\right)\right\}_{m \in \mathbb{N}}$ of (LB)-spaces forms a projective spectrum and $\mathcal{O}_{M, \omega}\left(\mathbb{R}^{N}\right)=\cap_{m=1}^{\infty} \cup_{n=1}^{\infty} \mathcal{O}_{n, \omega}^{m}\left(\mathbb{R}^{N}\right)$. We denote by $t$ the projective topology on the space $\mathcal{O}_{M, \omega}\left(\mathbb{R}^{N}\right)$ defined by $\left\{\cup_{n=1}^{\infty} \mathcal{O}_{n, \omega}^{m}\left(\mathbb{R}^{N}\right)\right\}_{m \in \mathbb{N}}$. The next aim is to compare the topology $\tau$ with the topology $t$. To this end, we introduce the following spaces.

Definition 5.3 Let $\omega$ be a non-quasianalytic weight function. For $m \in \mathbb{N}$ we define the space

$$
\mathcal{O}_{s_{\omega}}^{m}\left(\mathbb{R}^{N}\right):=\left\{f \in C^{\infty}\left(\mathbb{R}^{N}\right): \forall g \in \mathcal{S}_{\omega}\left(\mathbb{R}^{N}\right) q_{m, g}(f)<\infty\right\}
$$

and endow it with the lc-topology $\tau_{m}$ generated by the system of norms $\left\{q_{m, g}\right\}_{g \in \mathcal{S}_{\omega}\left(\mathbb{R}^{N}\right)}$.

It is straightforward to verify that the following topological equality holds

$$
\left(\mathcal{O}_{M, \omega}\left(\mathbb{R}^{N}\right), \tau\right)=\cap_{m=1}^{\infty}\left(\mathcal{O}_{s_{\omega}}^{m}\left(\mathbb{R}^{N}\right), \tau_{m}\right)
$$

when the space on the right hand side is endowed with the corresponding projective limit topology. Moreover, the following results hold.

Proposition 5.4 Let $\omega$ be a non-quasianalytic weight function and $m \in \mathbb{N}$. Then the following properties are satisfied. 
(1) The inclusion $\cup_{n=1}^{\infty} \mathcal{O}_{n, \omega}^{m}\left(\mathbb{R}^{N}\right) \hookrightarrow\left(\mathcal{O}_{s_{\omega}}^{m}\left(\mathbb{R}^{N}\right), \tau_{m}\right)$ is well-defined and continuous.

(2) $\cup_{n=1}^{\infty} \mathcal{O}_{n, \omega}^{m}\left(\mathbb{R}^{N}\right)=\mathcal{O}_{s_{\omega}}^{m}\left(\mathbb{R}^{N}\right)$ algebraically. Moreover, the spaces $\cup_{n=1}^{\infty} \mathcal{O}_{n, \omega}^{m}\left(\mathbb{R}^{N}\right)$ and $\left(\mathcal{O}_{s_{\omega}}^{m}\left(\mathbb{R}^{N}\right), \tau_{m}\right)$ have the same bounded sets.

(3) $\left(\mathcal{O}_{s_{\omega}}^{m}\left(\mathbb{R}^{N}\right), \tau_{m}\right)$ is a complete lc-space.

Proof (1) Fix $g \in \mathcal{S}_{\omega}\left(\mathbb{R}^{N}\right)$. Then for each $n \in \mathbb{N}$ there exists $c_{n}>0$ such that for every $x \in \mathbb{R}^{N}$ we have

$$
|g(x)| \leq c_{n} \exp (-n \omega(x))
$$

This implies for every $n \in \mathbb{N}$ that the inclusion

$$
\mathcal{O}_{n, \omega}^{m}\left(\mathbb{R}^{N}\right) \hookrightarrow \mathcal{O}_{s_{\omega}}^{m}\left(\mathbb{R}^{N}\right)
$$

is well-defined and continuous. Indeed, for a fixed $n \in \mathbb{N}$, we obtain via (5.8) that

$$
\begin{aligned}
q_{m, g}(f) & =\sup _{x \in \mathbb{R}^{N}} \sup _{\alpha \in \mathbb{N}_{0}^{N}}\left|g(x) \| \partial^{\alpha} f(x)\right| \exp \left(-m \varphi_{\omega}^{*}\left(\frac{|\alpha|}{m}\right)\right) \\
& \leq c_{n} \sup _{x \in \mathbb{R}^{N}} \sup _{\alpha \in \mathbb{N}_{0}^{N}}\left|\partial^{\alpha} f(x)\right| \exp \left(-n \omega(x)-m \varphi_{\omega}^{*}\left(\frac{|\alpha|}{m}\right)\right)=c_{n} r_{m, n}(f) .
\end{aligned}
$$

Since $g \in \mathcal{S}_{\omega}\left(\mathbb{R}^{N}\right)$ is arbitrary, the continuity of the inclusion $\mathcal{O}_{n, \omega}^{m}\left(\mathbb{R}^{N}\right) \hookrightarrow \mathcal{O}_{s_{\omega}}^{m}\left(\mathbb{R}^{N}\right)$ follows.

Since $\cup_{n=1}^{\infty} \mathcal{O}_{n, \omega}^{m}\left(\mathbb{R}^{N}\right)$ is an (LB)-space, we deduce that the inclusion $\cup_{n=1}^{\infty} \mathcal{O}_{n, \omega}^{m}$ $\left(\mathbb{R}^{N}\right) \hookrightarrow\left(\mathcal{O}_{s_{\omega}}^{m}\left(\mathbb{R}^{N}\right), \tau_{m}\right)$ is continuous.

(2) By (1) above it suffices to show only that every bounded subset of $\left(\mathcal{O}_{s_{\omega}}^{m}\left(\mathbb{R}^{N}\right), \tau_{m}\right)$ is also a bounded subset of $\cup_{n=1}^{\infty} \mathcal{O}_{n, \omega}^{m}\left(\mathbb{R}^{N}\right)$. To this end, we fix a bounded subset $B$ of $\left(\mathcal{O}_{s_{\omega}}^{m}\left(\mathbb{R}^{N}\right), \tau_{m}\right)$. We would show that there exists $n_{0} \in \mathbb{N}$ such that $\sup _{f \in B} r_{m, n_{0}}(f)<$ $\infty$. If this is not the case, then $\sup _{f \in B} r_{m, n}(f)=\infty$ for every $n \in \mathbb{N}$. To get a contradiction, we proceed as follows.

For each $j \in \mathbb{N}$ let $K_{j}:=\bar{B}_{j}(0) \times\left\{\alpha \in \mathbb{N}_{0}^{N}:|\alpha| \leq j\right\}$. Then $\cup_{j \in \mathbb{N}} K_{j}=\mathbb{R}^{N} \times \mathbb{N}_{0}^{N}$. On the other hand, taking in account that the function $g_{0}(x):=\exp \left(-|x|^{2}\right)$, for $x \in \mathbb{R}^{N}$, belongs to $\mathcal{S}_{\omega}\left(\mathbb{R}^{N}\right)$ we have for every $f \in B$ and $n, j \in \mathbb{N}$ that

$$
\begin{aligned}
& \sup _{(x, \alpha) \in K_{j}}\left|\partial^{\alpha} f(x)\right| \exp \left(-n \omega(x)-m \varphi_{\omega}^{*}\left(\frac{|\alpha|}{m}\right)\right) \\
& \leq \sup _{(x, \alpha) \in K_{j}} \exp \left(|x|^{2}-n \omega(x)\right)\left|\partial^{\alpha} f(x)\right| g_{0}(x) \exp \left(-m \varphi_{\omega}^{*}\left(\frac{|\alpha|}{m}\right)\right) \leq k_{j} q_{m, g_{0}}(f),
\end{aligned}
$$

where $k_{j}:=\sup _{(x, \alpha) \in K_{j}} \exp \left(|x|^{2}-n \omega(x)\right)<\infty$, and hence

$$
\sup _{f \in B} \sup _{(x, \alpha) \in K_{j}}\left|\partial^{\alpha} f(x)\right| \exp \left(-n \omega(x)-m \varphi_{\omega}^{*}\left(\frac{|\alpha|}{m}\right)\right)<\infty .
$$


Since $\sup _{f \in B} r_{m, n}(f)=\infty$, an argument by induction then yields that there exist a sequence $\left\{f_{n}\right\}_{n \in \mathbb{N}} \subseteq B$ and a strictly increasing sequence $\left\{j_{n}\right\}_{n \in \mathbb{N}}$ of positive integers such that for every $n \in \mathbb{N}$ there exists some point $\left(x_{n}, \alpha_{n}\right) \in \stackrel{\circ}{K_{j_{n+1}}} \backslash K_{j_{n-1}}$ for which

$$
\left|\partial^{\alpha_{n}} f_{n}\left(x_{n}\right)\right| \exp \left(-n \omega\left(x_{n}\right)-m \varphi_{\omega}^{*}\left(\frac{|\alpha|}{m}\right)\right)>n
$$

Now, we choose a function $\rho \in \mathcal{D}_{\omega}\left(\mathbb{R}^{N}\right)$ such that $\operatorname{supp} \rho \subseteq B_{1}(0)$ and $\rho(0)=1$. We define

$$
g(x):=\sum_{k=1}^{\infty} \exp (-k \omega(x)) \rho\left(x-x_{k}\right), \quad x \in \mathbb{R}^{N} .
$$

Then by Lemma 4.2 we have $g \in \mathcal{S}_{\omega}\left(\mathbb{R}^{N}\right)$. In particular, $g\left(x_{n}\right)=\exp \left(-n \omega\left(x_{n}\right)\right)$ for every $n \in \mathbb{N}$. Hence, by (5.9) it follows for every $n \in \mathbb{N}$ that

$$
\begin{aligned}
q_{m, g}\left(f_{n}\right) & \geq g\left(x_{n}\right)\left|\partial^{\alpha_{n}} f_{n}\left(x_{n}\right)\right| \exp \left(-m \varphi_{\omega}^{*}\left(\frac{\left|\alpha_{n}\right|}{m}\right)\right) \\
& \geq \exp \left(-n \omega\left(x_{n}\right)\right)\left|\partial^{\alpha_{n}} f_{n}\left(x_{n}\right)\right| \exp \left(-m \varphi_{\omega}^{*}\left(\frac{\left|\alpha_{n}\right|}{m}\right)\right)>n .
\end{aligned}
$$

This shows that $\sup _{f \in B} q_{m, g}(f)=\infty$, which is a contradiction as $B$ is a bounded subset of $\left(\mathcal{O}_{s_{\omega}}^{m}\left(\mathbb{R}^{N}\right), \tau\right)$ and hence $\sup _{f \in B} q_{m, g}(f)<\infty$.

(3) follows as in the proof of Theorem 5.2(2).

Let $X=$ ind ${ }_{n \rightarrow} X_{n}$ be an (LB)-space with canonical inclusions $j_{n}: X_{n} \rightarrow X$ for each $n \in \mathbb{N}$. Recall that $X$ is called regular if every bounded subset of $X$ is contained and bounded in a step $X_{m}$ for some $m \in \mathbb{N}$. Every complete (LB)-space is regular, [17, (5) p.225]. Accordingly, Proposition 3.5 implies that the space $\cup_{n=1}^{\infty} \mathcal{O}_{n, \omega}^{m}\left(\mathbb{R}^{N}\right)$ is a regular (LB)-space for each $m \in \mathbb{N}$. On the other hand, Proposition 5.4 yields another proof of the regularity of the (LB)-spaces $\cup_{n=1}^{\infty} \mathcal{O}_{n, \omega}^{m}\left(\mathbb{R}^{N}\right)$.

Proposition 5.5 Let $\omega$ be a non-quasianalytic weight function and $m \in \mathbb{N}$. Then $\cup_{n=1}^{\infty} \mathcal{O}_{n, \omega}^{m}\left(\mathbb{R}^{N}\right)$ is a regular $(L B)$-space. Moreover, $\cup_{n=1}^{\infty} \mathcal{O}_{n, \omega}^{m}\left(\mathbb{R}^{N}\right)$ is the bornological space associated with the space $\left(\mathcal{O}_{s_{\omega}}^{m}\left(\mathbb{R}^{N}\right), \tau_{m}\right)$.

Proof The result follows from Proposition 5.4(2). Indeed, in the proof of Proposition $5.4(2)$ it has been established also that every bounded subset of $\left(\mathcal{O}_{s_{\omega}}^{m}\left(\mathbb{R}^{N}\right), \tau_{m}\right)$ is contained and bounded in the Banch space $\mathcal{O}_{n, \omega}^{m}\left(\mathbb{R}^{N}\right)$ for some $n \in \mathbb{N}$.

Further immediate consequences of Proposition 5.4 are the following results.

Proposition 5.6 Let $\omega$ be a non-quasianalytic weight function. Then the inclusion

$$
\left(\mathcal{O}_{M, \omega}\left(\mathbb{R}^{N}\right), t\right) \hookrightarrow\left(\mathcal{O}_{M, \omega}\left(\mathbb{R}^{N}\right), \tau\right)
$$


is continuous, i.e., the topology $\tau$ is coarser than the topology $t$. Moreover, the spaces $\left(\mathcal{O}_{M, \omega}\left(\mathbb{R}^{N}\right), t\right)$ and $\left(\mathcal{O}_{M, \omega}\left(\mathbb{R}^{N}\right), \tau\right)$ have the same bounded subsets.

Proof Since

$$
\begin{aligned}
& \left(\mathcal{O}_{M, \omega}\left(\mathbb{R}^{N}\right), t\right)=\text { proj } m \leftarrow \cup_{n=1}^{\infty} \mathcal{O}_{n, \omega}^{m}\left(\mathbb{R}^{N}\right) \\
& \text { and } \left.\left(\mathcal{O}_{M, \omega}\left(\mathbb{R}^{N}\right), \tau\right)=\operatorname{proj} m \leftarrow\left(\mathcal{O}_{s_{\omega}}^{m}\left(\mathbb{R}^{N}\right), \tau_{m}\right)\right),
\end{aligned}
$$

the result follows immediately from Proposition 5.4(1)-(2).

Proposition 5.7 Let $\omega$ be a non-quasianalytic weight function. Then the inclusion

$$
\mathcal{S}_{\omega}\left(\mathbb{R}^{N}\right) \hookrightarrow\left(\mathcal{O}_{M, \omega}\left(\mathbb{R}^{N}\right), \tau\right)
$$

is continuous with dense range.

Proof The result immediately follows from Proposition 5.6, Theorems 3.8 and 3.9.

Let $X$ be a Hausdorff lc-space and $\Gamma_{X}$ be a system of continuous seminorms generating the topology of $X$. Then the strong operator topology $\tau_{s}$ in the space $\mathcal{L}(X)$ of all continuous linear operators from $X$ into itself is determined by the family of seminorms $q_{x}(S):=q(S x)(S \in \mathcal{L}(X))$ for each $x \in X$ and $q \in \Gamma_{X}$. In such a case we write $\mathcal{L}_{S}(X)$. Denoted by $\mathcal{B}(X)$ the collection of all bounded subsets of $X$, the topology $\tau_{b}$ of uniform convergence on bounded sets is defined in $\mathcal{L}(X)$ by the seminorms $q_{B}(S):=\sup _{x \in B} q(S x)(S \in \mathcal{L}(X))$ for each $B \in \mathcal{B}(X)$ and $q \in \Gamma_{X}$. In such a case we write $\mathcal{L}_{b}(X)$.

By Theorem 4.4 the space $\mathcal{O}_{M, \omega}\left(\mathbb{R}^{N}\right)$ can be identified with the space $\mathcal{M}\left(\mathcal{S}_{\omega}\left(\mathbb{R}^{N}\right)\right)$ of all multipliers on $\mathcal{S}_{\omega}\left(\mathbb{R}^{N}\right)$ via the map $M: \mathcal{O}_{M, \omega}\left(\mathbb{R}^{N}\right) \rightarrow \mathcal{M}\left(\mathcal{S}_{\omega}\left(\mathbb{R}^{N}\right)\right)$ defined by $M(f):=M_{f}$ for each $f \in \mathcal{O}_{M, \omega}\left(\mathbb{R}^{N}\right)$. Since $\mathcal{M}\left(\mathcal{S}_{\omega}\left(\mathbb{R}^{N}\right)\right)$ is a subspace of $\mathcal{L}\left(\mathcal{S}_{\omega}\left(\mathbb{R}^{N}\right)\right)$, the space $\mathcal{O}_{M, \omega}\left(\mathbb{R}^{N}\right)$ (via the map $M$ ) can be then endowed with either the topology $\tau_{b}$ induced by $\mathcal{L}_{b}\left(\mathcal{S}_{\omega}\left(\mathbb{R}^{N}\right)\right)$ or the topology $\tau_{s}$ induced by $\mathcal{L}_{s}\left(\mathcal{S}_{\omega}\left(\mathbb{R}^{N}\right)\right)$. In the next result we compare the three topologies $\tau_{b}, \tau_{s}$ and $\tau$.

To this end, we first show the following variant of Lemma 4.2.

Lemma 5.8 Let $\omega$ be a non-quasianalytic weight function. Let $h: \mathbb{R}^{N} \rightarrow \mathbb{R}$ be a non-negative function satisfying the condition

$$
\forall \lambda>0 \quad \lim _{|x| \rightarrow \infty} \exp (\lambda \omega(x)) h(x)=0
$$

Then there exists $g \in \mathcal{S}_{\omega}\left(\mathbb{R}^{N}\right)$ such that

$$
\forall x \in \mathbb{R}^{N} \quad h(x) \leq g(x) .
$$

Proof Let $\rho \in \mathcal{D}_{\omega}\left(\mathbb{R}^{N}\right)$ such that $\rho \geq 0, \rho \equiv 1$ on $B_{1}(0)$ and $\operatorname{supp} \rho \subseteq B_{1}(0)$. Let $\left\{x_{j}\right\}_{j \in \mathbb{N}} \subseteq \mathbb{R}^{N}$ be a sequence satisfying the following properties: $\lim _{j \rightarrow \infty}\left|x_{j}\right|=\infty$; there exists $H \in \mathbb{N}$ such that $\left|\left\{j \in \mathbb{N}: x \in B_{2}\left(x_{j}\right)\right\}\right| \leq H$ for every $x \in \mathbb{R}^{N}$; for any $x \in \mathbb{R}^{N}$ there exists $j \in \mathbb{N}$ such that $x \in B_{1}\left(x_{j}\right)$. 
Let

$$
a_{j}:=\sup _{x \in \bar{B}_{2}\left(x_{j}\right)} h(x), \quad j \in \mathbb{N},
$$

and

$$
g(x):=\sum_{j \in \mathbb{N}} a_{j} \rho\left(x-x_{j}\right), \quad x \in \mathbb{R}^{N},
$$

where the series on the right of (5.16) is a finite sum for every $x \in \mathbb{R}^{N}$.

For any fixed $x \in \mathbb{R}^{N}$, let $j \in \mathbb{N}$ such that $x \in B_{1}\left(x_{j}\right)$. Then $g(x) \geq a_{j} \geq h(x)$. This shows that $h(x) \leq g(x)$ for all $x \in \mathbb{R}^{N}$. So, it remains to establish that $g \in$ $\mathcal{S}_{\omega}\left(\mathbb{R}^{N}\right)$.

Since the property $(\gamma)$ of $\omega$ implies that $\exp (\omega(x)) \geq \exp (a)(1+|x|)^{b}$ for every $x \in \mathbb{R}^{N}$, by [9, Lemma 3.6, p.127] we can conclude that $g \in \mathcal{S}\left(\mathbb{R}^{N}\right)$. In order to conclude the proof, we proceed as follows.

Fix $\lambda, \mu>0$ and $x \in \mathbb{R}^{N}$. Then we have for every $\alpha \in \mathbb{N}_{0}^{N}$ that

$$
\partial^{\alpha} g(x)=\sum_{x \in \bar{B}_{2}\left(x_{j}\right)} a_{j} \partial^{\alpha} \rho\left(x-x_{j}\right)
$$

where the set $J(x):=\left\{j \in \mathbb{N}: x \in \bar{B}_{2}\left(x_{j}\right)\right\}$ has cardinality less or equal to that of $H$, with $H$ indipendent of $x$. Since $\rho \in \mathcal{D}_{\omega}\left(\mathbb{R}^{N}\right)$, it follows for every $\alpha \in \mathbb{N}_{0}^{N}$ that

$$
\begin{aligned}
& \exp (\mu \omega(x))\left|\partial^{\alpha} g(x)\right| \leq \\
& \quad \leq H \sup _{j \in J(x)} a_{j} \exp (\mu \omega(x)) \exp \left(-\mu K \omega\left(x-x_{j}\right)+\lambda \varphi_{\omega}^{*}\left(\frac{|\alpha|}{\lambda}\right)\right) q_{\lambda, \mu K}(\rho),
\end{aligned}
$$

where $K$ is the constant appearing in the property $(\alpha)$ of $\omega$. On the other hand, by (5.13) there exists $C>0$ such that $h(x) \leq C \exp \left(-\mu K^{2} \omega(x)\right)$ for all $x \in \mathbb{R}^{N}$ and so, we have for every $j \in \mathbb{N}$ that

$$
a_{j}=\sup _{x \in \bar{B}_{2}\left(x_{j}\right)} h(x)=\sup _{y \in \bar{B}_{2}(0)} h\left(y+x_{j}\right) \leq C \sup _{y \in \bar{B}_{2}(0)} \exp \left(-\mu K^{2} \omega\left(y+x_{j}\right)\right) .
$$

But, the following inequalities are satisfied

$$
\omega(x)=\omega\left(\left(x-x_{j}\right)+x_{j}\right) \leq K\left(\omega\left(x-x_{j}\right)+\omega\left(x_{j}\right)+1\right)
$$

and

$$
\omega\left(x_{j}\right)=\omega\left(\left(y+x_{j}\right)-y\right) \leq K\left(\omega\left(y+x_{j}\right)+\omega(y)+1\right) .
$$


Accordingly, by (5.17) and (5.18) it follows for every $\alpha \in \mathbb{N}_{0}^{N}$ that

$$
\begin{aligned}
\exp (\mu \omega(x))\left|\partial^{\alpha} g(x)\right| \leq & H C \sup _{j \in J(x)} \sup _{y \in \bar{B}_{2}(0)} \exp \left(K^{2} \mu(\omega(y)+1)\right) \exp \left(-K \mu \omega\left(x_{j}\right)\right) \\
& \times e^{\mu K} \exp \left(\mu K \omega\left(x-x_{j}\right)\right) \exp \left(\mu K \omega\left(x_{j}\right)\right) \exp \left(-\mu K \omega\left(x-x_{j}\right)\right. \\
& \left.+\lambda \varphi_{\omega}^{*}\left(\frac{|\alpha|}{\lambda}\right)\right) q_{\lambda, \mu K}(\rho) \\
& =H C e^{\mu K} \sup _{y \in \bar{B}_{2}(0)} \exp \left(K^{2} \mu(\omega(y)+1)\right) \exp \left(\lambda \varphi_{\omega}^{*}\left(\frac{|\alpha|}{\lambda}\right)\right) q_{\lambda, \mu K}(\rho)
\end{aligned}
$$

and hence,

$$
\exp \left(-\lambda \varphi_{\omega}^{*}\left(\frac{|\alpha|}{\lambda}\right)\right) \exp (\mu \omega(x))\left|\partial^{\alpha} g(x)\right| \leq H C e^{\mu K} \sup _{y \in \bar{B}_{2}(0)} \exp \left(K^{2} \mu(\omega(y)+1)\right) q_{\lambda, \mu K}(\rho) .
$$

Since $D:=\sup _{y \in \bar{B}_{2}(0)} \exp \left(K^{2} \mu(\omega(y)+1)\right)<\infty$ is a constant independent of $x$ and $x$ is arbitrary, we can conclude that

$$
q_{\lambda, \mu}(g) \leq H C D e^{\mu K} q_{\lambda, \mu K}(\rho)<\infty .
$$

But, $\lambda$ and $\mu$ are also arbitrary. So, this implies that $g \in \mathcal{S}_{\omega}\left(\mathbb{R}^{N}\right)$.

Theorem 5.9 Let $\omega$ be a non-quasianalytic weight function. Then the inclusions

$$
\left(\mathcal{O}_{M, \omega}\left(\mathbb{R}^{N}\right), \tau\right) \hookrightarrow\left(\mathcal{O}_{M, \omega}\left(\mathbb{R}^{N}\right), \tau_{b}\right) \hookrightarrow\left(\mathcal{O}_{M, \omega}\left(\mathbb{R}^{N}\right), \tau_{s}\right)
$$

are continuous. Moreover, the spaces $\left(\mathcal{O}_{M, \omega}\left(\mathbb{R}^{N}\right), \tau\right), \quad\left(\mathcal{O}_{M, \omega}\left(\mathbb{R}^{N}\right), \tau_{b}\right)$ and $\left(\mathcal{O}_{M, \omega}\left(\mathbb{R}^{N}\right), \tau_{s}\right)$ have the same bounded subsets.

Proof Since $\tau_{s} \subseteq \tau_{b}$, it suffices to show that $\tau_{b} \subseteq \tau$. To this end, let $W$ be a 0 neighbourhood of $\mathcal{L}_{b}\left(\mathcal{S}_{\omega}\left(\mathbb{R}^{N}\right)\right)$. Then there exist a 0-neighbourhood $V$ of $\mathcal{S}_{\omega}\left(\mathbb{R}^{N}\right)$ and a bounded subset $B$ of $\mathcal{S}_{\omega}\left(\mathbb{R}^{N}\right)$ such that

$$
\left\{T \in \mathcal{L}\left(\mathcal{S}_{\omega}\left(\mathbb{R}^{N}\right)\right): T(B) \subseteq V\right\} \subseteq W
$$

We can suppose that $V=\left\{h \in \mathcal{S}_{\omega}\left(\mathbb{R}^{N}\right): q_{m, n}(f) \leq \epsilon\right\}$ for some $m, n \in \mathbb{N}$ and $\epsilon>0$. To conclude the proof, we have to show that there exists a 0 -neighbourhood $U$ of $\left(\mathcal{O}_{M, \omega}\left(\mathbb{R}^{N}\right), \tau\right)$ such that $f g \in V$ for all $f \in U$ and $g \in B$. To this end, let $M \in \mathbb{N}$ such that $M \geq L m$ and $\epsilon^{\prime}>0$ such that $\epsilon^{\prime}<\epsilon e^{-m L}$, where $L \geq 1$ is the constant appearing in formula (2.5). Then the set $U$ of all functions $f \in \mathcal{O}_{M, \omega}\left(\mathbb{R}^{N}\right)$ satisfying the condition

$$
\sup _{x \in \mathbb{R}^{N}} \sup _{\gamma, \delta \in \mathbb{N}_{0}^{N}} \exp \left(n \omega(x)-M \varphi_{\omega}^{*}\left(\frac{|\gamma+\delta|}{M}\right)\right)\left|\partial^{\gamma} f(x)\right|\left|\partial^{\delta} g(x)\right| \leq \epsilon^{\prime}, \quad g \in B,
$$


is a 0 -neighbourhood of $\left(\mathcal{O}_{M, \omega}\left(\mathbb{R}^{N}\right), \tau\right)$ for which $f g \in V$ for all $f \in U$ and $g \in B$. Indeed, for fixed $f \in U$ and $g \in B$, we have for every $\alpha \in \mathbb{N}_{0}^{N}$ and $x \in \mathbb{R}^{N}$ that

$$
\begin{aligned}
\left|\partial^{\alpha}(f g)(x)\right| & \leq \sum_{\gamma \leq \alpha}\left(\begin{array}{l}
\alpha \\
\gamma
\end{array}\right)\left|\partial^{\gamma} f(x) \| \partial^{\alpha-\gamma} g(x)\right| \leq \epsilon^{\prime} \sum_{\gamma \leq \alpha}\left(\begin{array}{l}
\alpha \\
\gamma
\end{array}\right) \exp \left(-n \omega(x)+M \varphi_{\omega}^{*}\left(\frac{|\alpha|}{M}\right)\right) \\
& \leq \epsilon^{\prime} 2^{|\alpha|} \exp (-n \omega(x)) \exp \left(M \varphi_{\omega}^{*}\left(\frac{|\alpha|}{M}\right)\right) \\
& \leq \epsilon^{\prime} e^{m L} \exp (-n \omega(x)) \exp \left(m \varphi_{\omega}^{*}\left(\frac{|\alpha|}{m}\right)\right) \\
& \leq \epsilon \exp \left(-n \omega(x)+m \varphi_{\omega}^{*}\left(\frac{|\alpha|}{m}\right)\right) .
\end{aligned}
$$

after having used inequality (2.6). Therefore, $q_{m, n}(f g) \leq \epsilon$ and so $f g \in V$.

It remains to show that $U$ is a 0 -neighbourhood of $\left(\mathcal{O}_{M, \omega}\left(\mathbb{R}^{N}\right), \tau\right)$. To see this, we define

$$
h(x):=\exp (n \omega(x)) \sup _{g \in B} \sup _{\delta \in \mathbb{N}_{0}^{N}} \exp \left(-M \varphi_{\omega}^{*}\left(\frac{|\delta|}{M}\right)\right)\left|\partial^{\delta} g(x)\right|, \quad x \in \mathbb{R}^{N}
$$

Since $B$ a bounded subset of $\mathcal{S}_{\omega}\left(\mathbb{R}^{N}\right)$, we have for every $\lambda \geq 0$ and $x \in \mathbb{R}^{N}$ that

$$
\begin{aligned}
\exp (\lambda \omega(x)) h(x) & \leq \exp ((\lambda+n) \omega(x)) \sup _{g \in B} \sup _{\delta \in \mathbb{N}_{0}^{N}} \exp \left(-M \varphi_{\omega}^{*}\left(\frac{|\delta|}{M}\right)\right)\left|\partial^{\delta} g(x)\right| \\
& \leq \sup _{g \in B} q_{M, \lambda+n}(g)<\infty
\end{aligned}
$$

Accordingly, $h$ is a well-defined non-negative function on $\mathbb{R}^{N}$ satisfying condition (5.13). So, by Lemma 5.8 there exists $g_{1} \in \mathcal{S}_{\omega}\left(\mathbb{R}^{N}\right)$ such that $h(x) \leq g_{1}(x)$ for all $x \in \mathbb{R}^{N}$. Then $U^{\prime}:=\left\{f \in \mathcal{O}_{M, \omega}\left(\mathbb{R}^{N}\right): q_{M, g_{1}}(f) \leq \epsilon^{\prime}\right\}$ is a 0-neighbourhood of $\left(\mathcal{O}_{M, \omega}\left(\mathbb{R}^{N}\right), \tau\right)$. Moreover, for fixed $f \in U^{\prime}$ and $g \in B$, we have for every $\gamma, \delta \in \mathbb{N}_{0}^{N}$ that

$$
\begin{aligned}
\left|\partial^{\gamma} f(x)\right|\left|\partial^{\delta} g(x)\right| & \leq\left|\partial^{\gamma} f(x)\right| g_{1}(x) \exp (-n \omega(x)) \exp \left(M \varphi_{\omega}^{*}\left(\frac{|\delta|}{M}\right)\right) \\
& \leq \epsilon^{\prime} \exp \left(M \varphi_{\omega}^{*}\left(\frac{|\gamma|}{M}\right)\right) \exp (-n \omega(x)) \exp \left(M \varphi_{\omega}^{*}\left(\frac{|\delta|}{M}\right)\right) \\
& =\epsilon^{\prime} \exp (-n \omega(x)) \exp \left(M \varphi_{\omega}^{*}\left(\frac{|\gamma|}{M}\right)+M \varphi_{\omega}^{*}\left(\frac{|\delta|}{M}\right)\right) \\
& \leq \epsilon^{\prime} \exp (-n \omega(x)) \exp \left(M \varphi_{\omega}^{*}\left(\frac{|\gamma+\delta|}{M}\right)\right) .
\end{aligned}
$$

Since $f \in U^{\prime}$ and $g \in B$ are arbitrary, this implies that $U^{\prime} \subseteq U$. So, $U$ is a 0 neighbourhood of $\left(\mathcal{O}_{M, \omega}\left(\mathbb{R}^{N}\right), \tau\right)$. 
In order to show that the spaces $\left(\mathcal{O}_{M, \omega}\left(\mathbb{R}^{N}\right), \tau\right),\left(\mathcal{O}_{M, \omega}\left(\mathbb{R}^{N}\right), \tau_{b}\right)$ and $\left(\mathcal{O}_{M, \omega}\left(\mathbb{R}^{N}\right), \tau_{s}\right)$ have the same bounded sets, it suffices to prove only that every bounded subset of $\left(\mathcal{O}_{M, \omega}\left(\mathbb{R}^{N}\right), \tau_{s}\right)$ is also a bounded subset of $\left(\mathcal{O}_{M, \omega}\left(\mathbb{R}^{N}\right), \tau\right)$. To this end, we fix a bounded subset $B$ of $\left(\mathcal{O}_{M, \omega}\left(\mathbb{R}^{N}\right), \tau_{S}\right)$ and suppose that $B$ is not a bounded subset of $\left(\mathcal{O}_{M, \omega}\left(\mathbb{R}^{N}\right), \tau\right)$. Then there exist $g \in \mathcal{S}_{\omega}\left(\mathbb{R}^{N}\right)$ and $m \in \mathbb{N}$ such that

$$
\sup _{f \in B} q_{m, g}(f)=\infty
$$

To get a contradiction, we first observe that the inclusion $\left(\mathcal{O}_{M, \omega}\left(\mathbb{R}^{N}\right), \tau_{s}\right) \hookrightarrow \mathcal{E}_{\omega}\left(\mathbb{R}^{N}\right)$ is also continuous. Indeed, fixed any compact subset $K$ of $\mathbb{R}^{N}$, let $h \in \mathcal{D}_{\omega}\left(\mathbb{R}^{N}\right)$ such that $h \equiv 1$ on $K$. Then $\partial^{\alpha}(f h)=\partial^{\alpha} f$ on $K$ for each $f \in \mathcal{O}_{M, \omega}\left(\mathbb{R}^{N}\right)$ and $\alpha \in \mathbb{N}_{0}^{N}$. This implies for every $f \in \mathcal{O}_{M, \omega}\left(\mathbb{R}^{N}\right)$ and $l \in \mathbb{N}$ that

$$
\begin{aligned}
p_{K, m}(f) & =\sup _{x \in K} \sup _{\alpha \in \mathbb{N}_{0}^{N}}\left|\partial^{\alpha} f(x)\right| \exp \left(-l \varphi_{\omega}^{*}\left(\frac{|\alpha|}{l}\right)\right) \\
& =\sup _{x \in K} \sup _{\alpha \in \mathbb{N}_{0}^{N}}\left|\partial^{\alpha}(f h)(x)\right| \exp \left(\omega(x)-l \varphi_{\omega}^{*}\left(\frac{|\alpha|}{l}\right)\right) \exp (-\omega(x)) \\
& \leq C_{K} q_{l, 1}(f h)
\end{aligned}
$$

where $C_{K}:=\sup _{x \in K} \exp (-\omega(x))<\infty$ is a positive constant depending only on $K$. Accordingly, the inclusion $\left(\mathcal{O}_{M, \omega}\left(\mathbb{R}^{N}\right), \tau_{s}\right) \hookrightarrow \mathcal{E}_{\omega}\left(\mathbb{R}^{N}\right)$ is continuous.

Let $K_{j}:=\bar{B}_{j}(0) \times\left\{\alpha \in \mathbb{N}_{0}^{N}:|\alpha| \leq j\right\}$ for each $j \in \mathbb{N}\left(\right.$ so, $\left.\cup_{j \in \mathbb{N}} K_{j}=\mathbb{R}^{N} \times \mathbb{N}_{0}^{N}\right)$. The continuity of the inclusion $\left(\mathcal{O}_{M, \omega}\left(\mathbb{R}^{N}\right), \tau_{s}\right) \hookrightarrow \mathcal{E}_{\omega}\left(\mathbb{R}^{N}\right)$ implies for every $f \in B$ and $n, j \in \mathbb{N}$ that

$$
\begin{aligned}
& \sup _{(x, \alpha) \in K_{j}}\left|g(x) \| \partial^{\alpha} f(x)\right| \exp \left(-m \varphi_{\omega}^{*}\left(\frac{|\alpha|}{m}\right)\right) \leq \\
& \quad \leq k_{j} \sup _{(x, \alpha) \in K_{j}}\left|\partial^{\alpha} f(x)\right| \exp \left(-m \varphi_{\omega}^{*}\left(\frac{|\alpha|}{m}\right)\right) \leq k_{j} p_{\bar{B}_{j}(0), m}(f),
\end{aligned}
$$

where $k_{j}:=\sup _{x \in \bar{B}_{j}(0)}|g(x)|<\infty$, and hence

$$
\sup _{f \in B} \sup _{(x, \alpha) \in K_{j}}|g(x)|\left|\partial^{\alpha} f(x)\right| \exp \left(-m \varphi_{\omega}^{*}\left(\frac{|\alpha|}{m}\right)\right)<\infty .
$$

Taking in mind (5.21), we can then argue by induction to find a sequence $\left\{f_{n}\right\}_{n \in \mathbb{N}} \subseteq B$ and a strictly increasing sequence $\left\{j_{n}\right\}_{n \in \mathbb{N}}$ of positive integers such that for every $n \in \mathbb{N}$ there exists some point $\left(x_{n}, \alpha_{n}\right) \in \stackrel{\circ}{K}_{j_{n+1}} \backslash K_{j_{n-1}}$ for which

$$
\left|g\left(x_{n}\right)\right|\left|\partial^{\alpha_{n}} f_{n}\left(x_{n}\right)\right| \exp \left(-m \varphi_{\omega}^{*}\left(\frac{|\alpha|}{m}\right)\right)>n .
$$


Now, we choose a function $\rho \in \mathcal{D}_{\omega}\left(\mathbb{R}^{N}\right)$ such that $\operatorname{supp} \rho \subseteq B_{1}(0)$ and $\rho \equiv 1$ on $B_{r}(0)$ for some $0<r<1$. We define

$$
g_{0}(x):=\sum_{k=1}^{\infty} g\left(x_{k}\right) \rho\left(x-x_{k}\right), \quad x \in \mathbb{R}^{N} .
$$

Then by Remark 4.3 we have $g_{0} \in \mathcal{S}_{\omega}\left(\mathbb{R}^{N}\right)$. In particular, $g_{0} \equiv g\left(x_{n}\right)$ in $\bar{B}_{r}\left(x_{n}\right)$ for each $n \in \mathbb{N}$. Accordingly, for every $n \in \mathbb{N}, x \in \bar{B}_{r}\left(x_{n}\right)$ and $\alpha \in \mathbb{N}_{0}^{N}$ we have

$$
\partial^{\alpha}\left(f g_{0}\right)(x)=\partial^{\alpha} f(x) g\left(x_{n}\right) .
$$

Hence, by (5.23) it follows for every $n \in \mathbb{N}$ that

$$
\begin{aligned}
q_{m, m}\left(f_{n} g_{0}\right) & \geq\left|g\left(x_{n}\right)\right|\left|\partial^{\alpha_{n}} f_{n}\left(x_{n}\right)\right| \exp \left(m \omega(x)-m \varphi_{\omega}^{*}\left(\frac{\left|\alpha_{n}\right|}{m}\right)\right) \\
& \geq\left|g\left(x_{n}\right)\right|\left|\partial^{\alpha_{n}} f_{n}\left(x_{n}\right)\right| \exp \left(-m \varphi_{\omega}^{*}\left(\frac{\left|\alpha_{n}\right|}{m}\right)\right)>n .
\end{aligned}
$$

This shows that $\sup _{f \in B} q_{m, m}\left(f g_{0}\right)=\infty$, which is a contradiction because $B$ is a bounded subset of $\left(\mathcal{O}_{M, \omega}\left(\mathbb{R}^{N}\right), \tau_{s}\right)$. Hence $\sup _{f \in B} q_{m, g}(f)<\infty$.

Finally, we have

Proposition 5.10 Let $\omega$ be a non-quasianalytic weight function. Then $\left(\mathcal{O}_{M, \omega}\left(\mathbb{R}^{N}\right), \tau_{b}\right)$ and its strong dual are nuclear lc-spaces. Moreover, $\left(\mathcal{O}_{M, \omega}\left(\mathbb{R}^{N}\right), \tau_{b}\right)$ is complete.

Proof Since $\mathcal{S}_{\omega}\left(\mathbb{R}^{N}\right)$ is a nuclear Fréchet space by [5, Theorem 3.3], the space $\mathcal{L}_{b}\left(\mathcal{S}_{\omega}\left(\mathbb{R}^{N}\right)\right)$ and its strong dual space are nuclear lc-spaces, [12, Corollaire 3, Chap. II, §2, p.48]. Therefore, by [12, Théorème 9 , Chap. II, §2, p.47] $\left(\mathcal{O}_{M, \omega}\left(\mathbb{R}^{N}\right), \tau_{b}\right)$ and its strong dual are also nuclear lc-spaces.

It is straightforward to show the completeness of $\left(\mathcal{O}_{M, \omega}\left(\mathbb{R}^{N}\right), \tau_{b}\right)$.

Funding Open access funding provided by Universitá del Salento within the CRUI-CARE Agreement. This research is partially funded by GNAMPA.

Availability of data and material The manuscript has no associated data.

\section{Declarations}

Conflict of interest The author declares that she has no conflicts of interest regarding this study.

Code availability Not applicable.

Open Access This article is licensed under a Creative Commons Attribution 4.0 International License, which permits use, sharing, adaptation, distribution and reproduction in any medium or format, as long as you give appropriate credit to the original author(s) and the source, provide a link to the Creative Commons licence, 
and indicate if changes were made. The images or other third party material in this article are included in the article's Creative Commons licence, unless indicated otherwise in a credit line to the material. If material is not included in the article's Creative Commons licence and your intended use is not permitted by statutory regulation or exceeds the permitted use, you will need to obtain permission directly from the copyright holder. To view a copy of this licence, visit http://creativecommons.org/licenses/by/4.0/.

\section{References}

1. Beurling, A.: Quasi-analiticity and General Distributions. Lecture 4 and 5. AMS Summer Institute, Stanford (1961)

2. Björck, G.: Linear partial differential operators and generalized distributions. Ark. Mat. 6, 351-407 (1965)

3. Boiti, C., Jornet, D., Oliaro, A.: Real Paley-Wiener theorems in spaces of ultradifferentiable functions. J. Funct. Anal. 278, 1-45 (2020)

4. Boiti, C., Jornet, D., Oliaro, A.: Regularity of partial differential operators in ultradifferentiable spaces and Wigner type transforms. J. Math. Anal. Appl. 446, 920-944 (2017)

5. Boiti, C., Jornet, D., Oliaro, A., Schindl, G.: Nuclearity of rapidly decreasing ultradifferentiable functions and time-frequency analysis. Collect. Math. (2020). https://doi.org/10.1007/s13348-020-002960

6. Bonet, J., Meise, R., Melikhov, S.N.: A comparison of two different ways to define classes of ultradifferentiable functions. Bull. Belg. Math. Soc. Simon Stevin 14, 425-444 (2007)

7. Borwein, J..M., Lewis, A..S.: Convex Analysis and Nonlinear Optimization. Theory and Examples. CMS Books in Mathematics/Ouvrages de Mathématiques de la SMC, vol. 3. Springer, New York (2006)

8. Braun, R.W., Meise, R., Taylor, B.A.: Ultradifferentiable functions and Fourier analysis. Result. Math. 17, 206-237 (1990)

9. Chevalley, C.: Theory of Distributions: Lectures. Columbia University, New York (1951)

10. Dimovski, P., Prangoski, B., Velinov, D.: Multipliers and convolutors in the space of tempered ultradistrubutions. Novi Sad J. Math. 44, 1-18 (2014)

11. Gevrey, M.: Sur la nature analytique des solutions des equations aux dérivées partielles. Premier mémoire, Ann. Sci. Ec. Norm. Super. III. Ser. 35, 129-190 (1918)

12. Grothendieck, A.: Produits tensoriels topologiques et espaces nucléaires. Mem. Amer. Math. Soc. 16, (1955)

13. Horvath, J.: Topological Vector Spaces and Distributions, vol. 1. Addison-Wesley Publishing Company, Boston (1966)

14. Kaminski, A.: Convolution, product and Fourier transform of distribution. Stud. Math. 74, 83-96 (1982)

15. Kisynki, J.: Equicontinuity and Convergent Sequences in the Spaces $\mathcal{O}_{C}^{\prime}$ and $\mathcal{O}_{M}$. Bull. Polish Acad. Sci. Math. 59, 223-235 (2011)

16. Komatsu, H.: Ultradistributions 1. Structure theorems and a characterization. J. Fac. Sci. Tokyo Sec. IA 20, 25-105 (1973)

17. Köthe, G.: Topological Vector Spaces I, 2nd Rev. ed. Springer, Berlin (1983)

18. Kovacevic, D.: Some operations on the space $\mathcal{S}^{\prime}\left(M_{p}\right)$ of tempered ultradistributions. Univ. u Novom Sadu, Zb. Rad. Prirod.- Mat. Fak. Ser. Mat. 23, 87-106 (1993)

19. Kucera, J.: Convolution of temperate distributions. Publ. Math. Debrecen 33, 323-327 (1986)

20. Kucera, J., McKennon, K.: The topology on certain spaces of multipliers of temperate distributions. Rocky Mt. J. Math. 7, 377-383 (1977)

21. Larcher, J.: Multiplications and convolutions in L. Schwartz spaces of test functions and distributions and their continuity. Analysis (Int. J. Anal. Appl.) 33, 319-332 (2013)

22. Larcher, J., Wengenroth, J.: A new proof for the bornologicity of the space of slowly increasing functions. Bull. Belg. Math. Soc. Simon Stevin 21, 887-894 (2014)

23. Mujica, J.: A completeness criterion for inductive limits of Banach spaces. In: Functional Analysis, Holomorphy and Approximation Theory II. North-Holland Math. Stud., vol. 86, pp. 319-329 (1984)

24. Ortner, N., Wagner, P.: On the spaces $\mathcal{O}_{C}^{m}$ of John Horváth. J. Math. Anal. Appl. 415, 62-74 (2014) 
25. Pilipovic, S.: Multipliers, convolutors and hypoelliptic convolutors for tempered ultradistributions. In: Banaras Hindu University, India, Platinum Jubile Year International Symposium on Generalized Functions and Their Applications (December 23-26, 1991), Plenum Press, New York (1991)

26. Pilipovic, S.: Characterizations of bounded sets in spaces of ultradistributions. Proc. Am. Math. Soc. 12, 1191-1206 (1994)

27. Schwartz, L.: Théorie des Distributions. Hermann, Paris (1966)

28. Zielezny, Z.: On the space of convolution operators in $\mathcal{K}_{1}^{\prime}$. Stud. Math. 31, 111-124 (1968)

29. Zielezny, Z.: Hypoelliptic and entire elliptic convolution equations in subspaces of the space of distributions (II). Stud. Math. 32, 47-59 (1969)

Publisher's Note Springer Nature remains neutral with regard to jurisdictional claims in published maps and institutional affiliations. 